\title{
Determining the Number of Factors When the Number of Factors Can Increase with Sample Size
}

\author{
Hongjun Li \\ International School of Economics and Management, \\ Capital University of Economics and Business, Beijing, 100070, PR China \\ Qi Li \\ Department of Economics, Texas A\&M University \\ and \\ International School of Economics and Management, \\ Capital University of Economics and Business, Beijing, 100070, PR China \\ Yutang Shi* \\ School of Finance, Nankai University, Tianjin, 300350, PR China \\ and \\ Wells Fargo \& Company, USA
}

October 25, 2016

\begin{abstract}
Correctly specifying the number of factors $(r)$ is a fundamental issue for the application of factor models. In this paper we develop an econometric method to estimate the number of factors in factor models of large dimensions where the number of factors is allowed to increase as the two dimensions, cross-section size $(N)$ and time period $(T)$ increase. Using similar information criteria as proposed by Bai and $\mathrm{Ng}$ (2002), we show that the number of factors can be consistently estimated using the criteria. We propose a new procedure that avoids over estimating the number of factors while allowing for one to search for possible number of factors over a wide range of positive integers so that it also avoids underestimation of the number of factors. We conduct Monte-Carlo simulation to investigate the finite sample properties of the proposed approach.
\end{abstract}

JEL classication: C2, C3, C5, G1

Keywords: Principal Components, Factor Analysis, Increasing Number of Factors, Information Criteria.

\footnotetext{
${ }^{*}$ We would like to thank two anonymous referees, an associate editor and seminar participants at Princeton University, University of Southern California, University of Kansas, Xiamen University and Texas A\&M University for helpful comments that greatly improved our paper. Hongjun Li's research is partially supported by China National Science Foundation, project \# 71601130. The corresponding author: Yutang Shi: shij-2001@hotmail.com (emails for Qi Li: qi-li@tamu.edu and for Hongjun Li: econlee@163.com)
}

(C) 2016. This manuscript version is made available under the Elsevier user license http://www.elsevier.com/open-access/userlicense/1.0/ 


\section{Introduction}

Factor models have been widely used in economic analyses such as forecasting economic variables, estimating variance-covariance matrices with high dimensional data, and estimating average treatment effects, among others. In practice a few common factors may capture the variations of a large number of economic variables. In the finance literature, the arbitrage pricing theory (APT) of Ross (1976) assumes that a small number of factors can be used to explain a large number of asset returns. Stock and Watson $(1998,1999)$ consider forecasting inflation with diffusion indices ("factors") constructed from a large number of macroeconomic series. Gregory and Head (1999) and Forni, Hallin, Lippi, and Reichlin (2000) find that cross country variations have common components. Fan, Liao and Mincheva (2011), and Fan, Liao and Mincheva (2013) use factor models to estimate high dimensional variance-covariance matrices. Factor models can also be used to evaluate the impacts of various policies (e.g., Hsiao, Ching and Wan (2012)). By assuming that the cross-sectional correlations for all the units are attributed to the presence of some (unobserved) common factors, Hsiao, Ching and Wan (2012), Ching, Hsiao and Wan (2012), Bai, Li and Ouyang (2014), Ouyang and Peng (2015) and Li and Bell (2016) use panel data methods to construct the counterfactuals and to measure average treatment effects of some policy interventions based on factor models.

A fundamental issue of factor models is the correct specification of the number of factors, $r$. When the number of factors is fixed, Bai and Ng (2002), Onatski (2009), Ahn and Horenstein (2013), among others, have developed various approaches to consistently estimate the number of factors. But many empirical findings suggest that the number of factors may increase as the dimensions of the data $N$ increases, or $T$ increases. For many empirical analyses, the estimated number of factors ranges from one to more than ten, see Ludvigson and Ng (2009), Giannone, Reichlin and Sala (2005) and Forni and Gambetti (2010). This suggests that the number of factors may depend on sample sizes. One reason that the number of factors may increase with sample size is structural break, new factors may emerge after economic environments change. Using Bai and Ng's (2002) information criteria, Ludvigson and Ng (2007) find that the factor structure of their financial dataset comprising of $172(N=172)$ series quarterly financial indicators spanning the first quarter of 1960 through the fourth quarter of $2002(T=172)$ can be well described by $8(r=8)$ common factors. Jurado, Ludvigson and Ng (2013) update monthly version of the 147 financial time series used in Ludvigson and $\mathrm{Ng}$ (2007) and combine them with an updated version of 132 monthly macroeconomic series used in Ludvigson and $\mathrm{Ng}(2010)$. They find that $12(r=12)$ common factors can capture the variations of this new dataset with 279 series $(N=279)$ spanning the period 1959:01-2011:12 $(T=636)$.

Hence, Ludvigson and Ng's (2013) finding supports the argument that the number of factors may increase 
as sample size increases.

Assuming that the number of factors $r$ is fixed, there are many papers in the literature analyzing the problem of determining the number of factors. Some of them not only fix the number of factors, but also impose restrictions on the dimensions $N$ and $T$, such as Lewbel (1991), Donald (1997), Cragg and Donald (1997), Connor and Korajczyk (1993), Forni and Reichlin (1998) and Stock and Watson (1998). Imposing no restriction on the relation between $N$ and $T$ except that both $N$ and $T$ are assumed to be large, Bai and $\mathrm{Ng}$ (2002) treat the determination of the number of factors as a model selection problem, they propose some criteria and show that the number of factors can be consistently estimated by minimizing the proposed criteria. To improve the finite sample performances of Bai and Ng's (2002) criteria, Hallin and Liska (2007), and Alessi, Barigozzi and Capasso (2010) propose some modifications to Bai and Ng's (2002) procedure by introducing a tuning multiplicative constant in the penalty objective function. Onatski (2009) develops a test of the null of $k_{0}$ factors against the alternative that the number of factors $r$ satisfies $k_{0}<r \leq k_{1}$ for some finite positive integer $k_{1}$. Onatski also describes the asymptotic distribution of the test statistic with critical values tabulated. Onatski (2010) suggests to determine the number of factors from empirical distribution of eigenvalues of sample covariance matrix. Ahn and Horenstein (2013) exploit the fact that the $r$ largest eigenvalues of the variance matrix of $N$ response variables grow unboundedly as $N$ increases, while the other eigenvalues remain bounded to estimate the number of factors. All of the above mention works consider the case of a fixed number of factors ( $r$ is fixed). The main difference between our paper and the existing work is that we consider the problem of determining the number of factors in a factor model where the number of factors is allowed to increase as $N$ or $T$ increases.

Specifically, this paper is designed to provide an approach which enables one to estimate the number of factors consistently when the number of factors is allowed to increase as $N, T \rightarrow \infty$. We extend the method of Bai and Ng (2002) to penalize the number of factors with a penalty function which is determined by the sample sizes, $N$ and $T$, as well as the maximum possible number of factors allowed in the estimation. As the factors are unobserved, the estimation procedure takes two steps. First, assuming the number of factors to be an arbitrary number $1 \leq k \leq k_{\max }$, we estimate the factors $\left(\widehat{F}^{k}\right)$ using the principal components method, where $k_{\max }=k_{\max , N, T}$ is the maximum number for possible number of factors, which is assumed to be greater or equal to the true number of factors, whose value is determined by $N$ and $T$ and it increases as $N, T$ increases. Second, we select the number of factors $\hat{k}$ by minimizing a criterion modified from Bai and $\mathrm{Ng}(2002)$, which is a function of $k$ and the estimated factors $\left(\widehat{F}^{k}\right)$. This criterion depends on the usual trade-off between good fit and parsimony. We show that this method produces a consistent estimator of the number of factors $r$. However, simulation results show that the selected number of factor $\hat{k}$ can be sensitive 
to the choice of $k_{\max }$ and it tends to choose a $\hat{k}$ that is larger than $r$ when $k_{\max }$ is large. We propose using a new ('mode' based) selection procedure to overcome this problem so that the selected $\hat{k}$ is not sensitive to different $k_{\max }$ values used in practice.

The rest of this paper is organized as follows. Section 2 sets up the model and presents the assumptions associated with the model. Section 3 presents the estimating procedures and the theoretical properties of the proposed estimators. Section 4 reports simulation experiments to examine the finite sample performances of our proposed method when $r$ increases with $N$ or $T$. Concluding remarks are given in Section 5 . All the proofs are given in the Appendix.

\section{Factor Models}

We consider the problem of determining the number of factors $(r)$ in a static approximate factor model, allowing $r=r_{N, T} \rightarrow \infty$, as $N \rightarrow \infty$, or $T \rightarrow \infty$, or both $N, T \rightarrow \infty$, but with a slower rate than $\min \{N, T\}$, i.e., $\max \{r / N, r / T\} \rightarrow 0$, as $N, T \rightarrow \infty$.

Let $X_{i t}$ denote the response variable for unit $i$ at time t, for $i=1, \ldots, N$, and $t=1, \ldots, T$. Our model is of the following form

$$
X_{i t}=\frac{1}{\sqrt{r}} \lambda_{i}^{0^{\prime}} F_{t}^{0}+e_{i t}
$$

where $F_{t}^{0}$ is an $r \times 1$ vector of common factors, $\lambda_{i}^{0}$ is the $r \times 1$ vector of factor loadings, and $e_{i t}$ is the idiosyncratic error of the response variable $X_{i t}$. The factors, factor loadings and idiosyncratic errors are not observed. Without loss of generality, we can assume that $E\left(X_{i t}\right)=0$. If this is not the case, we can de-mean the data first.

Note that at the right-hand-side of our model (1), we divide $\lambda_{i}^{0^{\prime}} F_{t}^{0}$ by $\sqrt{r}$. This is because we allow for $r$ to diverge when $N, T \rightarrow \infty$. If we do not divide $\lambda_{i}^{0^{\prime}} F_{t}^{0}$ by $\sqrt{r}$, then the variance of the systematic part, $\lambda_{i}^{0^{\prime}} F_{t}^{0}$, is proportional to $r$ and the variance of idiosyncratic error $e_{i t}$ is finite, the variance of noise part over the variance of information part will go to zero, or equivalently, the goodness-of-fit $R^{2}$ will converge to one. By dividing $\lambda_{i}^{0^{\prime}} F_{t}^{0}$ by $\sqrt{r}$, we have $\operatorname{Var}\left(r^{-1 / 2} \lambda_{i}^{0^{\prime}} F_{t}^{0}\right)=O(1)$ and we can obtain a reasonable goodness-of-fit that is not too close to one.

Let $\operatorname{tr}(A)$ denote the trace of a square matrix $A$. The norm of a matrix $A$ is defined as $\|A\|=\left[\operatorname{tr}\left(A^{\prime} A\right)\right]^{1 / 2}$. We use $M_{1}$ to denote a generic positive constant and use $\mathcal{N}$ to denote the set of natural number. We make the main assumptions as follows:

Assumption A (Factors and loadings):

1. For all $t, r^{-2} E\left\|F_{t}^{0}\right\|^{4}<M_{1}$; 
2. There exists a $r \times r$ positive definite matrix $\Sigma_{F}$ such that $\left\|T^{-1} \sum_{t=1}^{T} F_{t}^{0} F_{t}^{0^{\prime}}-\Sigma_{F}\right\| \stackrel{p}{\rightarrow} 0$ as $T \rightarrow \infty$;

3. $\max _{1 \leq i \leq N} r^{-2} E\left\|\lambda_{i}^{0}\right\|^{4} \leq M_{1}<\infty$

4. Let $\Lambda^{0}$ be the $N \times r$ factor loading matrix with its $i^{t h}$ row given by $\lambda_{i}^{0}$. Then there exists a $r \times r$ positive definite matrix $D$ such that $\left\|N^{-1} \Lambda^{0^{\prime}} \Lambda^{0}-D\right\| \stackrel{p}{\rightarrow} 0$ as $N \rightarrow \infty$;

5. Let $\lambda_{i l}^{0}$ and $F_{t l}^{0}$ be the $l^{t h}$ components $(l=1, \ldots, r)$ of $\lambda_{i}^{0}$ and $F_{t}^{0}$, respectively. Then for all $(i, t)$, $E\left\{\left[r^{-1 / 2} \sum_{l=1}^{r} E\left(\lambda_{i l}^{0} F_{t l}^{0}\right)\right]^{4}\right\} \leq M_{1}$

Assumption B (Idiosyncratic Components): As $N, T \rightarrow \infty$ and $0<\lim _{N, T \rightarrow \infty} \frac{T}{N}<\infty$,

1. For all $i$ and $t, E\left(e_{i t}\right)=0, E\left|e_{i t}\right|^{8} \leq M_{1}$;

2. $E\left(N^{-1} e_{s}^{\prime} e_{t}\right)=E\left(N^{-1} \sum_{i=1}^{N} e_{i s} e_{i t}\right)=\gamma_{N}(s, t),\left|\gamma_{N}(s, s)\right| \leq M_{1}$ for all $s$, and that $T^{-1} \sum_{s=1}^{T} \sum_{t=1}^{T}\left|\gamma_{N}(s, t)\right| \leq M_{1}$

3. $E\left(e_{i t} e_{j t}\right)=\tau_{i j, t}$ with $\left|\tau_{i j, t}\right| \leq\left|\tau_{i j}\right|$ for some $\tau_{i j}$ and for all $t$; furthermore, $N^{-1} \sum_{i=1}^{N} \sum_{j=1}^{N}\left|\tau_{i j}\right| \leq M_{1}$;

4. $E\left(e_{i t} e_{j s}\right)=\tau_{i j, t s}$ and $(N T)^{-1} \sum_{i=1}^{N} \sum_{j=1}^{N} \sum_{t=1}^{T} \sum_{s=1}^{T}\left|\tau_{i j, t s}\right| \leq M_{1}$;

5. for every $(t, s), E\left|N^{-1 / 2} \sum_{i=1}^{N}\left[e_{i s} e_{i t}-E\left(e_{i s} e_{i t}\right)\right]\right|^{4} \leq M_{1}$;

6. We assume that there exist a $T \times T$ matrix $L$, a $N \times N$ matrix $R$, and a $T \times N$ matrix $\varepsilon$ such that

$$
e=L \varepsilon R
$$

where $L(T \times T)$ and $R(N \times N)$ are arbitrary non-random positive definite matrices, and $\varepsilon=\left(\varepsilon_{t i}\right)$ is a $T \times N$ matrix consisting of independent elements with uniformly bounded $7^{t h}$ moment and $E\left(\varepsilon_{i t}\right)=0$.

\section{Assumption C}

1. Weak Dependence Between Factors and Idiosyncratic Components:

$$
E\left(\frac{1}{N} \sum_{i=1}^{N}\left\|\frac{1}{\sqrt{T r}} \sum_{t=1}^{T} F_{t}^{0} e_{i t}\right\|^{2}\right) \leq M_{1}
$$

2. Weak Dependence Between Factor Loadings and Idiosyncratic Components:

$$
E\left(\frac{1}{T} \sum_{t=1}^{N}\left\|\frac{1}{\sqrt{N r}} \sum_{i=1}^{N} e_{i t} \lambda_{i}^{0}\right\|^{2}\right) \leq M_{1} .
$$

Conditions in Assumption A are modified from Assumptions A-B in Bai and Ng (2002) by taking care of the fact that $r \rightarrow \infty$ as $N, T \rightarrow \infty$. It is easy to see that assumption A1 holds true if $E\left[\left(F_{t l}^{0}\right)^{4}\right]=$ 
$O(1)$ for all $t=1, \ldots, T$ and for all $l=1, \ldots, r$. A2 imposes a restriction on the rate of $r$. For example with $\Sigma_{F}=T^{-1} \sum_{t=1}^{T} E\left(F_{t}^{0} F_{t}^{0^{\prime}}\right)$, it can be easily shown that A2 holds true if $r=o\left(T^{1 / 2}\right)$ and $\sum_{t=1}^{T} \sum_{s \neq t}^{T} \operatorname{Cov}\left(F_{t l}^{0} F_{t m}^{0}, F_{s l}^{0} F_{s m}^{0}\right)=O(T)$ for all $l, m \in\{1, \ldots, r\}$. This is because $E\left[\| T^{-1} \sum_{t=1}^{T} F_{t}^{0} F_{t}^{0^{\prime}}\right.$ $\left.-\Sigma_{F} \|^{2}\right]=T^{-2} \sum_{t=1}^{T} \sum_{s=1}^{T} \sum_{l=1}^{r} \sum_{m=1}^{r} E\left\{\left[F_{t l}^{0} F_{t m}^{0}-E\left(F_{t l}^{0} F_{t m}^{0}\right)\right]\left[F_{s l}^{0} F_{s m}^{0}-E\left(F_{s l}^{0} F_{s m}^{0}\right)\right]\right\}=O\left(r^{2} / T\right)$ if $\sum_{t=1}^{T} \sum_{s \neq t}^{T}$ $E\left\{\left[F_{t l}^{0} F_{t m}^{0}-E\left(F_{t l}^{0} F_{t m}^{0}\right)\right]\left[F_{s l}^{0} F_{s m}^{0}-E\left(F_{s l}^{0} F_{s m}^{0}\right)\right]\right\}=O(T)$ for all $l, m=1, \ldots, r$. Similarly, A3 holds true if $E\left[\left(\lambda_{i l}^{0}\right)^{4}\right]=O(1)$ for all $i=1, \ldots, N$ and for all $l=1, \ldots, r$. A4 is similar to A2, it holds true with $D=$ $N^{-1} \sum_{i=1}^{N} E\left(\lambda_{i}^{0} \lambda_{i}^{0^{\prime}}\right)$, if $r=o\left(N^{1 / 2}\right)$ and $\sum_{i=1}^{N} \sum_{j \neq i}^{T} \operatorname{Cov}\left(\lambda_{i l}^{0} \lambda_{j m}^{0}, \lambda_{j l}^{0} \lambda_{j m}^{0}\right)=O(N)$ for all $l, m \in\{1, \ldots, r\}$. A5 requires that $\lambda_{i l}^{0} F_{t l}^{0}$ is a weakly dependent process in $l$ because we allow for $r \rightarrow \infty$.

Conditions in Assumption B are basically the same as Assumption C in Bai and Ng (2002) because the idiosyncratic error $e_{i t}$ is unrelated to $r$ whether $r$ is finite or is allowed to diverge to infinity with the sample size. In particular, B5 is similar to A5 in that it assumes that, for all $(t, s), e_{i t} e_{i s}$ is a weakly dependent process in $i$. Assumption B6 puts a structure on the idiosyncratic components. This structure allows heteroscedasticity in both the time and cross-section dimensions, and also limited autocorrelation and cross-sectional correlation in the components.

Finally, assumption C is similar to assumption D in Bai and $\mathrm{Ng}$ (2002) except that we modified it by dividing the quantity by $\sqrt{r}$ as $r$ is allowed to diverge as $N$ and $T$ tend to infinity. They allow for limited time-series and cross-section dependence in idiosyncratic component and also weak dependence between factors (factor loadings) and idiosyncratic errors.

\section{Estimating the Common Factors and the Number of Factors}

Following Bai and $\mathrm{Ng}$ (2002), we estimate the common factor in a large panel by the principal components method. For $k \in\left\{1, \ldots, k_{\max }\right\}$, where $k_{\max }$ is allowed to increase at a slower speed than $\min \{N, T\}$ such that $k_{\max }=o\left(\min \left\{N^{1 / 3}, T\right\}\right)$. Let $\lambda_{i}^{k}$ and $F_{t}^{k}$ denote $k \times 1$ vectors of the loadings and factors with the allowance of $k$ factors in the estimation. The method of principal components minimizes

$$
V(k)=\min _{\Lambda^{k}, F^{k}} \frac{1}{N T} \sum_{i=1}^{N} \sum_{t=1}^{T}\left(X_{i t}-\frac{1}{\sqrt{k}} \lambda_{i}^{k^{\prime}} F_{t}^{k}\right)^{2}
$$

over $1 \leq k \leq k_{\max }$, subject to the normalization of either $\Lambda^{k^{\prime}} \Lambda^{k} / N=I_{k}$ or $F^{k^{\prime}} F^{k} / T=I_{k}$, where $\Lambda^{k}$ and $F^{k}$ are the $N \times k$ and $T \times k$ factor loading and factor matrices, respectively.

Let $\operatorname{ev}_{(i)}(A)$ denote the $i^{\text {th }}$ largest eigenvalue of matrix $A$, and $\mathrm{EV}_{(i)}(A)$ is the eigenvector corresponding to the eigenvalue $\operatorname{ev}_{(\mathrm{i})}(A)$ of the matrix $A$. If one concentrates out $\Lambda^{k}$ and uses the normalization that $F^{k^{\prime}} F^{k} / T=I_{k}$. The estimated factor matrix is $\tilde{F}^{k}=\sqrt{T}\left(\mathrm{EV}_{(1)}(X X)^{\prime}, \ldots, \operatorname{EV}_{(k)}\left(X X^{\prime}\right)\right)$. Given $\tilde{F}^{k}$, $\tilde{\Lambda}^{k^{\prime}}=\sqrt{k}\left(\tilde{F}^{k^{\prime}} \tilde{F}^{k}\right)^{-1} \tilde{F}^{k^{\prime}} X=\sqrt{k} \tilde{F}^{k^{\prime}} X / T$ is the corresponding matrix of factor loadings. On the other 
hand, if one concentrates out $F^{k}$ and uses the normalization that $\Lambda^{k^{\prime}} \Lambda^{k} / N=I_{k}$, the solution to the above problem is given by $\left(\bar{F}^{k}, \bar{\Lambda}^{k}\right)$, where $\bar{\Lambda}^{k}=\sqrt{N}\left(\mathrm{EV}_{(1)}\left(X^{\prime} X\right), \ldots, \mathrm{EV}_{(k)}\left(X^{\prime} X\right)\right)$. The normalization that $\Lambda^{k^{\prime}} \Lambda^{k} / N=I_{k}$ implies $\bar{F}^{k}=\sqrt{k} X \bar{\Lambda}^{k} / N$.

Define $\widehat{F}^{k}=\bar{F}^{k}\left(\bar{F}^{k^{\prime}} \bar{F}^{k} / T\right)^{1 / 2}$, a rescaled estimator of the factors. This rescaled estimator has the asymptotic properties summarized in the following theorem.

Proposition 3.1 Under the assumptions $A-C$, for any $1 \leq k \leq k_{\max }=o\left(\min \left\{N^{1 / 3}, T\right\}\right)$ there exists a $(r \times k)$ matrix $H^{k}$ with $\mathrm{rank}=\min \{k, r\}$ such that

$$
\frac{1}{T} \sum_{t=1}^{T}\left\|\hat{F}_{t}^{k}-H^{k^{\prime}} F_{t}^{0}\right\|^{2}=O_{p}\left(\max \left\{\frac{k^{3} r}{N}, \frac{k^{3}}{T}\right\}\right) .
$$

Similar to the results of Bai and Ng (2002), Proposition 3.1 suggests that the time average of the squared deviations between the estimated factors $\widehat{F}^{k}$ and those that lie in the true factor space, $H^{k^{\prime}} F_{t}^{0}$, will vanish as $N, T \rightarrow \infty$. However, the convergence rate depends on not only the panel structure $N$ and $T$, but also the factor structure $r$ and $k$.

Given the results of Proposition 3.1, we can now analyze the problem of determining the number of factors. Let $V\left(k, F^{k}\right)=\min _{\Lambda} \frac{1}{N T} \sum_{i=1}^{N} \sum_{t=1}^{T}\left(X_{i t}-\frac{1}{\sqrt{k}} \lambda_{i}^{k^{\prime}} F_{t}^{k}\right)^{2}$ be the sum of squared residuals (divided by $N T$ ), where the residuals are from regression models of regressing $X_{i}$ on the $k$ factors for all $i=1, \ldots, N$, and $X_{i}=\left(X_{i 1}, X_{i 2}, \ldots, X_{i T}\right)^{\prime}$ is a $T \times 1$ vector of time-series observations for the $i$ th cross-section unit. The selecting criterion modified from those suggested by Bai and Ng (2002) has the form

$$
P C(k)=V\left(k, \widehat{F}^{k}\right)+k g(N, T)
$$

where $g(N, T)$ is the penalty factor satisfying two conditions: (i) $k_{\max } \cdot g(N, T) \rightarrow 0$ as $N, T \rightarrow \infty$, (ii) $C_{N, T, k_{\max }}^{-1} g(N, T) \rightarrow \infty$ as $N, T \rightarrow \infty$, where $C_{N, T, k_{\max }}=O_{p}\left(\max \left\{\frac{k_{\max }^{3}}{\sqrt{N}}, \frac{k_{\text {max }}^{5 / 2}}{\sqrt{T}}\right\}\right)$. As $V\left(k, \widehat{F}^{k}\right)$ is decreasing in $k$, the criterion above penalizes $k$ with a penalty factor $k g(N, T)$ to select the estimator $\hat{k}$ such that asymptotically under and overparameterized models will not be chosen. Theorem 3.1 formally establishes this result.

Theorem 3.1 Let $1 \leq r \leq k_{\text {max }}=o\left(\min \left\{N^{1 / 17}, T^{1 / 16}\right\}\right)$ and $\hat{k}=\operatorname{argmin}_{1 \leq k \leq k_{\max }} P C(k)$. Suppose that Assumptions $A$-C hold, and that (i) $k_{\max } \cdot g(N, T) \rightarrow 0$, (ii) $C_{N, T, k_{\max }}^{-1} \cdot g(N, T) \rightarrow \infty$ as $N, T \rightarrow \infty$. Then

$$
\lim _{N, T \rightarrow \infty} \operatorname{Prob}[\hat{k}=r]=1
$$


A formal proof of Theorem 3.1 is provided in the Appendix. Conditions (i) and (ii) together define the type of penalty factor that should vanish at an appropriate rate. They are sufficient conditions for estimation consistency so that they may not always be required for consistent estimating the number of factors.

Remark 3.1 Since we often need to divide some quantities by $r$, we rule out the case that $r=0$. Allowing for $r=0$ in our framework will complicate the regularity conditions, notations and proofs. Therefore, we did not consider the case that $r=0$ in our paper. The $r=0$ case is covered in Bai and Ng's (2002). Their procedure can be used to select the number of factors even when the true number of factors is 0 . We also conducted some simulations which show that both Bai and Ng's (2002) original method and the modified method proposed in our paper work well when $r=0$.

Note that the condition imposed in $k_{\max }$ is asymmetric in $(N, T)$. This result is induced by Proposition 3.1. The details can be found in the proof of Proposition 3.1 given in the appendix. As a referee correctly points out, if in the proof of Theorem 3.1, instead of using the result of Proposition 3.1 that $T^{-1} \sum_{t=1}^{T}\left\|\hat{F}_{t}^{k}-H^{k^{\prime}} F_{t}^{0}\right\|^{2}=O_{p}\left(\max \left\{\frac{k^{3} r}{N}, \frac{k^{3}}{T}\right\}\right)$, one may use $N^{-1} \sum_{i=1}^{N}\left\|\hat{\lambda}_{i}^{k}-\tilde{H}^{k^{\prime}} \lambda_{i}^{0}\right\|^{2}=O_{p}\left(\max \left\{\frac{k^{3}}{N}, \frac{k^{3} r}{T}\right\}\right),{ }^{1}$ where $\tilde{H}^{k}$ is a $r \times k$ matrix with $\operatorname{rank}\left(\tilde{H}^{k}\right)=\min \{r, k\}$. Then the condition that $1 \leq r \leq k_{\max }=$ $o\left(\min \left\{N^{1 / 17}, T^{1 / 16}\right\}\right)$ in Theorem 3.1 will be replaced by $1 \leq r \leq k_{\max }=o\left(\min \left\{N^{1 / 16}, T^{1 / 17}\right\}\right)$. The result is still asymmetric in $N$ and $T$, but the roles of $N$ and $T$ are exchanged.

In fact, it is possible to obtain a symmetric result (of $k_{\max }$ in $N$ and $T$ ) under some stronger regularity conditions, i.e., one can obtain a symmetric condition for $k_{\max }$ as $1 \leq r \leq k_{\max }=o\left(\min \left\{N^{1 / 16}, T^{1 / 16}\right\}\right)$ in Theorem 3.1 under some stronger assumptions. We state this result in the following proposition.

Proposition 3.2 Under the same conditions as in Proposition 3.1 except that we strength some conditions as follows: (i) $\lambda_{i l}$ is non-random with $\lambda_{i l} \leq \bar{\lambda}<\infty$ for all $i=1, \ldots, N$ and $l=1, \ldots, r$; (ii) $E\left(e_{i t} e_{j t}\right)=0$ for all $t \in\{1, \ldots, T\}$ and for all $j \neq i, i, j \in\{1, \ldots, N\}, E\left(F_{t l}^{0} F_{t m}^{0}\right)=0$ for all $t \in\{1, \ldots, T\}$ and for all $m \neq l$, $l, m \in\{1, \ldots, r\}$; (iii) $e_{i t}$ and $F_{s}^{0}$ are independent with each other for all $i, t$ and $s$. Then

$$
\frac{1}{T} \sum_{t=1}^{T}\left\|\hat{F}_{t}^{k}-H^{k^{\prime}} F_{t}^{0}\right\|^{2}=O_{p}\left(k^{3}\left(\frac{1}{N}+\frac{1}{T}\right)\right) .
$$

The proof of Proposition 3.2 is given in the appendix. Under Proposition 3.2, the condition $1 \leq r \leq k_{\max }=$ $o\left(\min \left\{N^{1 / 17}, T^{1 / 16}\right\}\right)$ in Theorem 3.1 can be replaced by $1 \leq r \leq k_{\max }=o\left(\min \left\{N^{1 / 16}, T^{1 / 16}\right\}\right)$. That is, we obtain a condition on $k_{\max }$ that is symmetric in $N$ and $T$.

Remark 3.2 The zero correlation assumption on $F_{t l}$ used in Proposition 3.2 is quite strong. However, it can be replaced by some weakly dependence assumptions such as $\rho$-mixing or $\beta$-mixing processes with mixing coefficients decay to zero at certain rates. But this will make the presentation (regarding regularity conditions) as well as the proofs of Proposition 3.2 much longer. Therefore, we will not pursue a proof of Proposition 3.2 under weak regularity conditions in this paper.

\footnotetext{
${ }^{1}$ This result can be proved similar to the proof of Proposition 3.1, its proof is available from the authors upon request.
} 
Similar to Bai and Ng (2002) we have the following Corollary.

Corollary 3.1 Under the Assumptions of Theorem 3.1, if one replaces PC $(k)$ in Theorem 3.1 by the class of criterion defined by

$$
I C(k)=\ln \left(V\left(k, \widehat{F}^{k}\right)\right)+k g(N, T)
$$

then the conclusion of Theorem 3.1 holds true.

Corollary 3.1 states that the class of criterion $I C(k)$ can also be used to consistently estimate the number of factors in factor models where the number of factors possibly increases with the sample size.

Let $\widehat{\sigma}^{2}$ be a consistent estimate of $(N T)^{-1} \sum_{i=1}^{N} \sum_{t=1}^{T} E\left(e_{i t}\right)^{2}$. Bai and $\mathrm{Ng}$ (2002) generalize the $C_{p}$ criterion of Mallows (1973) and suggest three $P C_{p}$ criteria as follows:

$$
\begin{aligned}
P C_{p 1}(k) & =V\left(k, \widehat{F}^{k}\right)+k \cdot \widehat{\sigma}^{2}\left(\frac{N+T}{N T}\right) \ln \left(\frac{N T}{N+T}\right), \\
P C_{p 2}(k) & =V\left(k, \widehat{F}^{k}\right)+k \cdot \widehat{\sigma}^{2}\left(\frac{N+T}{N T}\right) \ln (\min \{N, T\}), \\
P C_{p 3}(k) & =V\left(k, \widehat{F}^{k}\right)+k \cdot \widehat{\sigma}^{2}\left(\frac{\ln (\min \{N, T\})}{\min \{N, T\}}\right) .
\end{aligned}
$$

It is easy to check that these criteria satisfy the two conditions for the penalty factor in Theorem 3.1 if $k_{\max }=o\left(\left[\ln \left(\frac{N T}{N+T}\right)\right]^{1 / 6}\right)$. The three criteria have different finite-sample properties while they are asymptotically equivalent. In applications, Bai and $\mathrm{Ng}(2002)$ suggest to replace $\hat{\sigma}^{2}$ with $V\left(k_{\max }, \widehat{F}^{k_{\max }}\right)=$ $(N T)^{-1} \sum_{i=1}^{N} \sum_{t=1}^{T} \hat{e}_{i t}^{2}$, where $\hat{e}_{i t}=X_{i t}-\frac{1}{\sqrt{k}} \widehat{\lambda}_{i}^{k_{\max }^{\prime}} \widehat{F}_{t}^{k_{\max }}$ for $i=1, \ldots, N$ and $t=1, \ldots, T$, the residuals for the linear regression of $X$ on $\widehat{F}^{k_{\max }}$. Thus, the number of factors estimated using these three criteria may be sensitive to the selection of $k_{\max }$. We will propose a method that avoids the sensitivity of selected $\hat{k}$ depending on $k_{\max }$.

Corollary 3.1 suggests the following three $I C_{p}$ criteria can also be used to select the number of factors:

$$
\begin{aligned}
& I C_{p 1}(k)=\ln \left(V\left(k, \widehat{F}^{k}\right)\right)+k \cdot\left(\frac{N+T}{N T}\right) \ln \left(\frac{N T}{N+T}\right), \\
& I C_{p 2}(k)=\ln \left(V\left(k, \widehat{F}^{k}\right)\right)+k \cdot\left(\frac{N+T}{N T}\right) \ln (\min \{N, T\}), \\
& I C_{p 3}(k)=\ln \left(V\left(k, \widehat{F}^{k}\right)\right)+k \cdot\left(\frac{\ln (\min \{N, T\})}{\min \{N, T\}}\right) .
\end{aligned}
$$

The main advantage of the three criteria given in (8) is that the scaling factor $\hat{\sigma}^{2}$ is automatically removed by the logarithmic transformation. We do not need to estimate $\sigma^{2}$ before selecting the number of factors. Therefore, the number of factors estimated using $I C_{p}$ criteria is insensitive to the selection of $k_{\max }$.

As the estimated $\hat{k}$ using $P C_{p}$ criteria may be sensitive to $k_{\max }$, the selection of $k_{\max }$ is an important issue in practice. Bai and $\mathrm{Ng}(2002)$ suggest to select $k_{\max }$ by setting $k_{\max }=8\left[(\min \{N, T\} / 100)^{1 / 4}\right]$ where 
$[A]$ denotes the integer part of a real number $A$. But their theoretical result does not cover this case as this $k_{\max }$ increases (without bound) with $N$ and $T$. Using some ad-hoc rules to select $k_{\max }$ may lead to $k_{\max }<r$, which will lead to an underestimation of the number of factors because if $k_{\max }<r$, then we will have $\hat{k} \leq k_{\max }<r$. On the other hand, if $k_{\max }$ is too large $\left(k_{\max }>>r\right)$, simulations show that the selected $\hat{k}$ tends to overestimate $r(\hat{k}>r)$. We propose a new procedure to resolve this problem. We propose to let $k_{\max }$ take a wide range of values. For each value of $k_{\max }$, we select a $\hat{k}_{k_{\max }}$ that minimizes the $P C_{p}$ criteria. We then select the value of $\hat{k}$ that appears most times among the different $\hat{k}_{k_{\max }}$ values, i.e., we select the mode of $\hat{k}_{k_{\max }}$ (over a wide range of $k_{\max }$ ). We use a specific example to illustrate this selection procedure. We generate a simulated data of $N=200, T=60$ with the true number of factors $r=7$. We let $k_{\max }$ take values from $\{1,2, \ldots, 40\}$. For each different $1 \leq k_{\max } \leq 40$, we select a $\hat{k}_{k_{\max }}$ by minimizing $P C_{p 1}$ criterion. The result is presented in Figure 1. From Figure 1 we observe that when $k_{\max }<r=7$, we select $\hat{k}=k_{\max }<7$ as expected; when $7 \leq k_{\max } \leq 16$, we select $\hat{k}=7$; when $k_{\max }>16$, the selected $\hat{k}>7$. Moreover, $\hat{k}$ increases with $k_{\max }$. We also notice that $\hat{k}=7$ is selected ten times (when $k_{\max }=7,8, \ldots, 16$ ), while all the other values are chosen no more than three times. For example, when $17 \leq k_{\max } \leq 19$, the selected $\hat{k}_{k_{\max }}=8$, i.e., $\hat{k}_{k_{\max }}=8$ is selected three times. According to our selection rule, $\hat{k}=7$ is selected because $\hat{k}=7$ appears most times (10 times).

Figure 2 plot $\hat{k}-k_{\max }$ curves for different $N, T$ and $r$ values. We see that although $\hat{k}$ increases with $k_{\max }$ for most cases, our proposed procedure can select the correct number of factors because $\hat{k}_{k_{\text {max }}}$ takes value $r$ more often than taking any other values for all cases reported in Figure 2. Hence, our proposed procedure of selecting $\hat{k}$ is not sensitive to $k_{\max }$ provided that one let $k_{\max }$ take a wide range of values. Therefore, we suggest letting $k_{\max }$ to take values in $\{1,2, \ldots,[6 \log (\max (N, T))]\}$ where $[A]$ denotes the integer part of a real number $A$. $[6 \log (\max (N, T))]$ is around 41, 45 and 55 when $\max (N, T)=1000,2000$ and 10000 . This setting is also consistent with our simulation since we let $r=[1.5 \log (\max (N, T))]$ in our simulations in section 4 .

\section{Simulations}

In this section we conduct Monte Carlo simulations to investigate how our modified criteria of Bai and $\mathrm{Ng}$ (2002) perform when the number of factors is allowed to increase with $N$ or $T$. For simplicity of the comparison with the simulation results in Bai and $\mathrm{Ng}$ (2002), we first fix $T$ and allow $N$ and $r$ to increase. When $T$ is fixed as 60 , we let $N=100,200,500,1000,2000$ and $r=[1.5 \log (N)]$, where $[A]$ denotes the integer part of a real number $A$; for $T=100$, we let $N=40,60,100,200,500,1000,2000$ and $r=[1.5 \log (N)]$. The simulation results for this case are reported in the upper part of each table for each data generating 
process (DGP). Next, we check the performance of the criteria when $\mathrm{N}$ is fixed and $\mathrm{T}$ keeps increasing. When $N=100$, we let $T=40,60,100,200,500,1000,2000$ and $r=[1.5 \log (T)]$; when $N=60$, we let $T=100,200,500,1000,2000$ and $r=[1.5 \log (T)]$. The simulation results for this case are reported in the lower part of each table for each DGP. We replicate the suggested estimating procedure 1000 times and the reported results are the averages of $\hat{k}$ over 1000 replications.

The data generating processes (DGP) have the following form:

$$
X_{i t}=\frac{1}{\sqrt{r}} \sum_{j=1}^{r} \lambda_{i j} F_{t j}+e_{i t},
$$

where $\lambda_{i j} \sim$ i.i.d. $N(0,1), F_{t j} \sim$ i.i.d. $N(0,2)$.

We consider three DGPs here. In the base case, we set the DGP as $e_{i t} \sim$ i.i.d.N $(0,1)$. This base DGP is denoted as DGP1. The simulation results for this case are reported in Table 1. We see that all information criterion give precise estimates of the number of factors.

Table 1: Estimated Number of Factors: DGP1

\begin{tabular}{ccccccccc}
\hline \hline$N$ & $T$ & $r$ & $P C_{p 1}$ & $P C_{p 2}$ & $P C_{p 3}$ & $I C_{p 1}$ & $I C_{p 2}$ & $I C_{p 3}$ \\
\hline 100 & 60 & 6 & 6 & 6 & 6 & 6 & 6 & 6 \\
200 & 60 & 7 & 7 & 7 & 7 & 7 & 7 & 7 \\
500 & 60 & 9 & 9 & 9 & 9 & 9 & 9 & 9 \\
1000 & 60 & 10 & 10 & 10 & 10 & 10 & 10 & 10 \\
2000 & 60 & 11 & 11 & 11 & 11 & 11 & 11 & 11 \\
40 & 100 & 5 & 5 & 5 & 5 & 5 & 5 & 5 \\
60 & 100 & 6 & 6 & 6 & 6 & 6 & 6 & 6 \\
100 & 100 & 6 & 6 & 6 & 6 & 6 & 6 & 6 \\
200 & 100 & 7 & 7 & 7 & 7 & 7 & 7 & 7 \\
500 & 100 & 9 & 9 & 9 & 9 & 9 & 9 & 9 \\
1000 & 100 & 10 & 10 & 10 & 10 & 10 & 10 & 10 \\
2000 & 100 & 11 & 11 & 11 & 11 & 11 & 11 & 11 \\
\hline \hline 100 & 40 & 5 & 5 & 5 & 5 & 5 & 5 & 5 \\
100 & 60 & 6 & 6 & 6 & 6 & 6 & 6 & 6 \\
100 & 100 & 6 & 6 & 6 & 6 & 6 & 6 & 6 \\
100 & 200 & 7 & 7 & 7 & 7 & 7 & 7 & 7 \\
100 & 500 & 9 & 9 & 9 & 9 & 9 & 9 & 9 \\
100 & 1000 & 10 & 10 & 10 & 10 & 10 & 10 & 10 \\
100 & 2000 & 11 & 11 & 11 & 11 & 11 & 11 & 11 \\
60 & 100 & 6 & 6 & 6 & 6 & 6 & 6 & 6 \\
60 & 200 & 7 & 7 & 7 & 7 & 7 & 7 & 7 \\
60 & 500 & 9 & 9 & 9 & 9 & 9 & 9 & 9 \\
60 & 1000 & 10 & 10 & 10 & 10 & 10 & 10 & 10 \\
60 & 2000 & 11 & 11 & 11 & 11 & 11 & 11 & 11 \\
\hline
\end{tabular}

DGP1: $X_{i t}=\frac{1}{\sqrt{r}} \sum_{j=1}^{r} \lambda_{i j} F_{t j}+e_{i t} ; r=[c * \ln (N)]$ for the upper part of the table, and $r=[c * \ln (T)]$ for the lower part, where $c=1.5$, and $[A]$ denotes the integer part of a real number $A$.

For the heterogeneity case of DGP2, we set the idiosyncratic shocks to be heterogeneous. We let $e_{i t}=$ 
Table 2: Estimated number of Factors: Heterogeneity

\begin{tabular}{ccccccccc}
\hline \hline$N$ & $T$ & $r$ & $P C_{p 1}$ & $P C_{p 2}$ & $P C_{p 3}$ & $I C_{p 1}$ & $I C_{p 2}$ & $I C_{p 3}$ \\
\hline 100 & 60 & 6 & 6 & 6 & 6 & $\mathbf{5}$ & $\mathbf{5}$ & 6 \\
200 & 60 & 7 & 7 & 7 & 7 & 7 & 7 & 7 \\
500 & 60 & 9 & 9 & 9 & 9 & $\mathbf{8}$ & $\mathbf{8}$ & $\mathbf{8}$ \\
1000 & 60 & 10 & 10 & 10 & 10 & 10 & 10 & 10 \\
2000 & 60 & 11 & 11 & 11 & 11 & 11 & 11 & 11 \\
40 & 100 & 5 & 5 & 5 & 5 & 5 & 5 & 5 \\
60 & 100 & 6 & 6 & 6 & 6 & 6 & 6 & 6 \\
100 & 100 & 6 & 6 & 6 & 6 & 6 & 6 & 6 \\
200 & 100 & 7 & 7 & 7 & 7 & 7 & 7 & 7 \\
500 & 100 & 9 & 9 & 9 & 9 & 9 & 9 & 9 \\
1000 & 100 & 10 & 10 & 10 & 10 & 10 & 10 & 10 \\
2000 & 100 & 11 & 11 & 11 & 11 & 11 & 11 & 11 \\
\hline 100 & 40 & 5 & 5 & 5 & 5 & 5 & 5 & 5 \\
100 & 60 & 6 & 6 & 6 & 6 & $\mathbf{5}$ & $\mathbf{5}$ & 6 \\
100 & 100 & 6 & 6 & 6 & 6 & 6 & 6 & 6 \\
100 & 200 & 7 & 7 & 7 & 7 & 7 & 7 & 7 \\
100 & 500 & 9 & 9 & 9 & 9 & 9 & 9 & 9 \\
100 & 1000 & 10 & 10 & 10 & 10 & 10 & 10 & 10 \\
100 & 2000 & 11 & 11 & 11 & 11 & 11 & 11 & 11 \\
60 & 100 & 6 & 6 & 6 & 6 & 6 & 6 & 6 \\
60 & 200 & 7 & 7 & 7 & 7 & 7 & 7 & 7 \\
60 & 500 & 9 & 9 & 9 & 9 & 9 & 9 & 9 \\
60 & 1000 & 10 & 10 & 10 & 10 & 10 & 10 & 10 \\
60 & 2000 & 11 & 11 & 11 & 11 & 11 & 11 & 11 \\
& & & & & & & &
\end{tabular}

DGP2: $X_{i t}=\frac{1}{\sqrt{r}} \sum_{j=1}^{r} \lambda_{i j} F_{t j}+e_{i t} ; e_{i t}=u_{i t}+\delta_{t} \epsilon_{i t}$, where $\delta_{t}=0$ for $t$ even, and $\delta_{t}=1$ for $t$ odd; $r=[c \ln (N)]$ for the upper part of the table, and $r=[c \ln (T)]$ for the lower part, where $[A]$ denotes taking the integer part of a real number.

$u_{i t}+\delta_{t} \epsilon_{i t}$ where $u_{i t} \sim$ i.i.d.N $(0,1), \epsilon_{i t} \sim i . i . d . N(0,1)$, and $\delta_{t}=0$ for even $t, \delta_{t}=1$ for odd $t$. Thus the variance of the idiosyncratic shocks is 1 when $t$ is odd and 2 when $t$ is even. We denote this DGP as DGP2. The estimated values of $\hat{k}$ are reported in Table 2 where the boldfaced numbers indicate incorrect selection of the number of factors. Similar to the homogeneous cases, $P C_{p 1}, P C_{p 2}$, and $P C_{p 3}$ perform well under all kinds of combinations of $N$ and $T$. The other three criteria $I C_{P 1}$ and $I C_{p 2}$, and $I C_{p 3}$ also perform well in general, although occasionally they may select $\hat{k}$ that is slightly smaller than the true number of factors $r$ when sample size is small.

For the last case, denoted as DGP3, we allow the idiosyncratic to be autocorrelated. We set $e_{i t}=$ $\rho e_{i t-1}+v_{i t}$, where $\rho=0.5$ and $v_{i t} \sim i . i . d . N(0,1)$. The estimation results are reported in Table 3 . The results for this case are almost the same as those of the base case except for $(N, T)=(60,200)$ with $r=7$, $I C_{p_{1}}$ and $I C_{p_{2}}$ select $r=6$. All other four information criteria perform quite well in accurately estimating the number of factors for all $(N, T)$ combinations for DGP3. 
Table 3: Estimated number of Factors: Autocorrelation

\begin{tabular}{ccccccccc}
\hline \hline$N$ & $T$ & $r$ & $P C_{p 1}$ & $P C_{p 2}$ & $P C_{p 3}$ & $I C_{p 1}$ & $I C_{p 2}$ & $I C_{p 3}$ \\
\hline 100 & 60 & 6 & 6 & 6 & 6 & 6 & 6 & 6 \\
200 & 60 & 7 & 7 & 7 & 7 & 7 & 7 & 7 \\
500 & 60 & 9 & 9 & 9 & 9 & 9 & 9 & 9 \\
1000 & 60 & 10 & 10 & 10 & 10 & 10 & 10 & 10 \\
2000 & 60 & 11 & 11 & 11 & 11 & 11 & 11 & 11 \\
40 & 100 & 5 & 5 & 5 & 5 & 5 & 5 & 5 \\
60 & 100 & 6 & 6 & 6 & 6 & 6 & 6 & 6 \\
100 & 100 & 6 & 6 & 6 & 6 & 6 & 6 & 6 \\
200 & 100 & 7 & 7 & 7 & 7 & 7 & 7 & 7 \\
500 & 100 & 9 & 9 & 9 & 9 & 9 & 9 & 9 \\
1000 & 100 & 10 & 10 & 10 & 10 & 10 & 10 & 10 \\
2000 & 100 & 11 & 11 & 11 & 11 & 11 & 11 & 11 \\
\hline \hline 100 & 40 & 5 & 5 & 5 & 5 & 5 & 5 & 5 \\
100 & 60 & 6 & 6 & 6 & 6 & 6 & 6 & 6 \\
100 & 100 & 6 & 6 & 6 & 6 & 6 & 6 & 6 \\
100 & 200 & 7 & 7 & 7 & 7 & 7 & 7 & 7 \\
100 & 500 & 9 & 9 & 9 & 9 & 9 & 9 & 9 \\
100 & 1000 & 10 & 10 & 10 & 10 & 10 & 10 & 10 \\
100 & 2000 & 11 & 11 & 11 & 11 & 11 & 11 & 11 \\
60 & 100 & 6 & 6 & 6 & 6 & 6 & 6 & 6 \\
60 & 200 & 7 & 7 & 7 & 7 & $\mathbf{6}$ & $\mathbf{6}$ & 7 \\
60 & 500 & 9 & 9 & 9 & 9 & 9 & 9 & 9 \\
60 & 1000 & 10 & 10 & 10 & 10 & 10 & 10 & 10 \\
60 & 2000 & 11 & 11 & 11 & 11 & 11 & 11 & 11 \\
\hline
\end{tabular}

DGP3: $X_{i t}=\frac{1}{\sqrt{r}} \sum_{j=1}^{r} \lambda_{i j} F_{t j}+e_{i t} ; e_{i t}=\rho e_{i t-1}+v_{i t} ; \rho=0.5 ; r=[c \ln (N)]$ for the upper part of the table, and $r=[c \ln (T)]$ for the lower part, where $[A]$ denotes taking the integer part of a real number.

Summarizing the results for all the DGPs we observe that $P C_{p 1}, P C_{p 2}$, and $P C_{p 3}$ have the best overall performance. $I C_{p} 1, I C_{p} 2$, and $I C_{p 3}$ perform well when the sample size is large $(\min \{N, T\}>100)$.

\section{Concluding Remarks}

In this paper, we consider the problem of determining the number of factors in large factor models where the number of factors is allowed to increase, but with a slower rate, as $N$ or $T$ increases. We extend the analysis of Bai and $\mathrm{Ng}(2002)$ to the case that number of factors can increase with the sample size and prove the consistency of a modified Bai and Ng's (2002) procedure in determining the number of factors. We also propose a ('mode' based) new procedure so that our selected number of factors is not sensitive to the choice of $k_{\max }$. Monte Carlo simulation results suggest that the criteria $P C_{p 1}, P C_{p 2}$ and $P C_{p 3}$ all have the overall best performance. Other criteria such as $I C_{p 1}, I C_{p 2}$ and $P C_{p 3}$ can also be used to accurately estimate the number of factors when the data dimensions are relatively large, say $\min \{N, T\} \geq 100$. One possible future research topic is to find alternative criteria that can improve the finite-sample performance of Bai and 
Ng's (2002) procedure and our modified procedure such that the new criteria can accurately determine the number of factors even in small or medium size samples. 


\section{Appendix A: Proofs}

\section{Proof of Proposition 3.1}

We will first prove a lemma (Lemma 1) below which will be used in proving Proposition 3.1.

Lemma 1 Under Assumptions A-C, we have for some positive constant $0<M_{2}<\infty$, and for all $N$ and $T$,

(i) $\frac{1}{T} \sum_{s=1}^{T} \sum_{t=1}^{T} \gamma_{N}(s, t)^{2} \leq M_{2}$;

(ii) $E\left(\frac{1}{T^{2}} \sum_{s=1}^{T} \sum_{t=1}^{T}\left(\frac{1}{N} \sum_{i=1}^{N} X_{i t} X_{i s}\right)^{2}\right) \leq M_{2}$;

Proof :

(i) See the proof of Lemma 1 (i) in Bai and $\mathrm{Ng}(2002)$.

(ii) If suffices to prove that for all $(i, t)$ that $E\left(X_{i t}^{4}\right) \leq M$. Now $E\left(X_{i t}^{4}\right) \leq 8 r^{-2} E\left[\left(\lambda_{i}^{0^{\prime}} F_{t}^{0}\right)^{4}\right]+8 E\left(e_{i t}^{4}\right) \leq 16 M_{1}$ by assumption A5 and B1.

Proof of Proposition 3.1:

Recall that $\hat{F}^{k}=\frac{\sqrt{k}}{N} X \tilde{\Lambda}^{k}$ and $\tilde{\Lambda}^{k}=\frac{\sqrt{k}}{T} X^{\prime} \tilde{F}^{k}$. From the normalization $\tilde{F}^{k^{\prime}} \tilde{F}^{k} / T=I_{k}$, we also have $(T k)^{-1} \sum_{t=1}^{T}\left\|\tilde{F}_{t}^{k}\right\|^{2}=1$. Following Bai and $\mathrm{Ng}(2002)$, using $H^{k^{\prime}}=\left(\tilde{F}^{k^{\prime}} F^{0} / T\right)\left(\Lambda^{0^{\prime}} \Lambda^{0} / N\right)$, we have

$$
\hat{F}_{t}^{k}-H^{k^{\prime}} F_{t}^{0}=\frac{k}{T} \sum_{s=1}^{T} \tilde{F}_{s}^{k} \gamma_{N}(s, t)+\frac{k}{T} \sum_{s=1}^{T} \tilde{F}_{s}^{k} \zeta_{s t}+\frac{k}{T} \sum_{s=1}^{T} \tilde{F}_{s}^{k} \eta_{s t}+\frac{k}{T} \sum_{s=1}^{T} \tilde{F}_{s}^{k} \xi_{s t},
$$

where $\zeta_{s t}=e_{s}^{\prime} e_{t} / N-\gamma_{N}(s, t), \eta_{s t}=F_{s}^{0^{\prime}} \Lambda^{0^{\prime}} e_{t} /(N \sqrt{r})$, and $\xi_{s t}=F_{t}^{0^{\prime}} \Lambda^{0^{\prime}} e_{s} /(N \sqrt{r})=\eta_{t s}$.

Because $(x+y+z+u)^{2} \leq 4\left(x^{2}+y^{2}+z^{2}+u^{2}\right),\left\|\hat{F}_{t}^{k}-H^{k^{\prime}} F_{t}^{0}\right\|^{2} \leq 4\left(a_{t}+b_{t}+c_{t}+d_{t}\right)$, where $a_{t}=$ $\frac{k^{2}}{T^{2}}\left\|\sum_{s=1}^{T} \tilde{F}_{s}^{k} \gamma_{N}(s, t)\right\|^{2}, b_{t}=\frac{k^{2}}{T^{2}}\left\|\sum_{s=1}^{T} \tilde{F}_{s}^{k} \zeta_{s t}\right\|^{2}, c_{t}=\frac{k^{2}}{T^{2}}\left\|\sum_{s=1}^{T} \tilde{F}_{s}^{k} \eta_{s t}\right\|^{2}$ and $d_{t}=\frac{k^{2}}{T^{2}}\left\|\sum_{s=1}^{T} \tilde{F}_{s}^{k} \xi_{s t}\right\|^{2}$. It follows that $(1 / T) \sum_{t=1}^{T}\left\|\hat{F}_{t}^{k}-H^{k^{\prime}} F_{t}^{0}\right\|^{2} \leq(4 / T) \sum_{t=1}^{T}\left(a_{t}+b_{t}+c_{t}+d_{t}\right)$.

By Cauchy's inequality, we have $\left\|\sum_{s=1}^{T} \tilde{F}_{s}^{k} \gamma_{N}(s, t)\right\|^{2} \leq\left(\sum_{s=1}^{T}\left\|\tilde{F}_{s}^{k}\right\|^{2}\right) \cdot\left(\sum_{s=1}^{T} \gamma_{N}(s, t)^{2}\right)$. Thus,

$$
\begin{aligned}
\frac{1}{T} \sum_{t=1}^{T} a_{t} & \leq \frac{k k^{2}}{T}\left(\frac{1}{T k} \sum_{s=1}^{T}\left\|\tilde{F}_{s}^{k}\right\|^{2}\right) \cdot \frac{1}{T}\left(\sum_{t=1}^{T} \sum_{s=1}^{T} \gamma_{N}(s, t)^{2}\right) \\
& =O_{p}\left(\frac{k^{3}}{T}\right)
\end{aligned}
$$

by Lemma 1(i) and the fact that $(T k)^{-1} \sum_{t=1}^{T}\left\|\tilde{F}_{t}^{k}\right\|^{2}=1$ (this follows from $\tilde{F}^{k^{\prime}} \tilde{F}^{k} / T=I_{k}$ ). 
For $b_{t}$, we have that

$$
\begin{aligned}
\frac{1}{T} \sum_{t=1}^{T} b_{t} & =\frac{k^{2}}{T^{3}} \sum_{t=1}^{T}\left\|\sum_{s=1}^{T} \tilde{F}_{s}^{k} \zeta_{s t}\right\|^{2} \\
& =\frac{k^{2}}{T^{3}} \sum_{t=1}^{T} \sum_{s=1}^{T} \sum_{u=1}^{T} \tilde{F}_{s}^{k^{\prime}} \tilde{F}_{u}^{k} \zeta_{s t} \zeta_{u t} \\
& \leq \frac{k^{2}}{T}\left(\frac{1}{T^{2}} \sum_{s=1}^{T} \sum_{u=1}^{T}\left(\tilde{F}_{s}^{k^{\prime}} \tilde{F}_{u}^{k}\right)^{2}\right)^{1 / 2}\left[\frac{1}{T^{2}} \sum_{s=1}^{T} \sum_{u=1}^{T}\left(\sum_{t=1}^{T} \zeta_{s t} \zeta_{u t}\right)^{2}\right]^{1 / 2} \\
& \leq \frac{k^{3}}{T}\left(\frac{1}{T k} \sum_{s=1}^{T}\left\|\tilde{F}_{s}^{k}\right\|^{2}\right)\left[\frac{1}{T^{2}} \sum_{s=1}^{T} \sum_{u=1}^{T}\left(\sum_{t=1}^{T} \zeta_{s t} \zeta_{u t}\right)^{2}\right]^{1 / 2} \\
& =k^{3}\left[\frac{1}{T^{4}} \sum_{s=1}^{T} \sum_{u=1}^{T}\left(\sum_{t=1}^{T} \zeta_{s t} \zeta_{u t}\right)^{2}\right]^{1 / 2} \\
& =O_{p}\left(\frac{k^{3}}{N}\right)
\end{aligned}
$$

where the last equality follows from $\left[\frac{1}{T^{4}} \sum_{s=1}^{T} \sum_{u=1}^{T}\left(\sum_{t=1}^{T} \zeta_{s t} \zeta_{u t}\right)^{2}\right]^{1 / 2}=O_{p}\left(N^{-1}\right)$ as shown in the proof of Theorem 1 of Bai and $\mathrm{Ng}$ (2002).

From $E\left(T^{-1} \sum_{t=1}^{T} \zeta_{s t} \zeta_{u t}\right)^{2}=E\left(T^{-2} \sum_{t=1}^{T} \sum_{v=1}^{T} \zeta_{s t} \zeta_{u t} \zeta_{s v} \zeta_{u v}\right) \leq \max _{s, t} E\left|\zeta_{s t}\right|^{4}$ and

$$
E\left|\zeta_{s t}\right|^{4}=\frac{1}{N^{2}} E\left|\frac{1}{\sqrt{N}} \sum_{i=1}^{N}\left(e_{i t} e_{i s}-E\left(e_{i t} e_{i s}\right)\right)\right|^{4} \leq \frac{1}{N^{2}} M_{1}
$$

by Assumption B5, we have

$$
\frac{1}{T} \sum_{t=1}^{T} b_{t} \leq O_{p}\left(k^{3}\right) \frac{1}{T} \sqrt{\frac{T^{2}}{N^{2}}}=O_{p}\left(\frac{k^{3}}{N}\right) .
$$

For $c_{t}$, we have

$$
\begin{aligned}
c_{t} & =\frac{k^{2}}{T^{2}}\left\|\sum_{s=1}^{T} \tilde{F}_{s}^{k} \eta_{s t}\right\|^{2} \\
& =\frac{k^{2}}{T^{2}}\left\|\sum_{s=1}^{T} \tilde{F}_{s}^{k} F_{s}^{0^{\prime}} \Lambda^{0^{\prime}} e_{t} / N \sqrt{r}\right\|^{2} \\
& \leq \frac{k^{2}}{N^{2}}\left\|e_{t}^{\prime} \Lambda^{0} / \sqrt{r}\right\|^{2}\left(\frac{k}{T k} \sum_{s=1}^{T}\left\|\tilde{F}_{s}^{k}\right\|^{2}\right)\left(\frac{r}{T r} \sum_{s=1}^{T}\left\|F_{s}^{0}\right\|^{2}\right) \\
& =\frac{k^{2}}{N^{2}}\left\|e_{t}^{\prime} \Lambda^{0} / \sqrt{r}\right\|^{2} O_{p}(k r)
\end{aligned}
$$

because $\frac{1}{T k} \sum_{s=1}^{T}\left\|\tilde{F}_{s}^{k}\right\|^{2}=1$ and $\frac{1}{T r} \sum_{s=1}^{T}\left\|F_{s}^{0}\right\|^{2}=O_{p}(1)$. 
It follows that

$$
\begin{aligned}
\frac{1}{T} \sum_{t=1}^{T} c_{t} & =O_{p}(k r) \frac{k^{2}}{N} \frac{1}{T} \sum_{t=1}^{T}\left\|\frac{e_{t}^{\prime} \Lambda^{0}}{\sqrt{N r}}\right\|^{2} \\
& =O_{p}\left(\frac{k^{3} r}{N}\right)
\end{aligned}
$$

because $\frac{1}{T} \sum_{t=1}^{T}\left\|\frac{e_{t}^{\prime} \Lambda^{0}}{\sqrt{N r}}\right\|^{2}=O_{p}(1)$ by assumption $\mathrm{C} 2$.

The term $(1 / T) \sum_{t=1}^{T} d_{t}=O_{p}\left(\frac{k^{3} r}{N}\right)$ can be proved similarly. Combining the above results, we have shown that

$$
\begin{aligned}
(1 / T) \sum_{t=1}^{T}\left\|\hat{F}_{t}^{k}-H^{k^{\prime}} F_{t}^{0}\right\|^{2} & \leq(4 / T) \sum_{t=1}^{T}\left(a_{t}+b_{t}+c_{t}+d_{t}\right) \\
& =O_{p}\left(\frac{k^{3} r}{N}\right)+O_{p}\left(\frac{k^{3}}{T}\right) .
\end{aligned}
$$

Alternatively, Proposition 3.1 can be proved by concentrating out $F_{t}$. Following the similar steps, we can show that

$$
\begin{aligned}
(1 / N) \sum_{i=1}^{N}\left\|\hat{\lambda}_{i}^{k}-H^{k^{\prime}} \lambda_{i}^{0}\right\|^{2} & \leq(4 / N) \sum_{i=1}^{N}\left(a_{i}+b_{i}+c_{i}+d_{i}\right) \\
& =O_{p}\left(\frac{k^{3}}{N}\right)+O_{p}\left(\frac{k^{3} r}{T}\right) .
\end{aligned}
$$

\section{Proof of Proposition 3.2}

Proof From the proof of Proposition 3.1 we know that

$$
\frac{1}{T} \sum_{t=1}^{T}\left\|\hat{F}_{t}^{k}-H^{k^{\prime}} F_{t}^{0}\right\|^{2} \leq(4 / T) \sum_{t=1}^{T}\left(a_{t}+b_{t}+c_{t}+d_{t}\right)
$$

and that $T^{-1} \sum_{t=1}^{T} a_{t}=O_{p}\left(k^{3} / T\right)$ and $T^{-1} \sum_{t=1}^{T} d_{t}=O_{p}\left(k^{3} / N\right)$. Therefore, we only need to show that $T^{-1} \sum_{t=1}^{T} c_{t}=O_{p}\left(k^{3} / N\right)$ and $T^{-1} \sum_{t=1}^{T} d_{t}=O_{p}\left(k^{3} / N\right)$. Since the proofs are similar. We will only prove for the term related to $c_{t}$.

For $c_{t}$, we have

$$
\begin{aligned}
c_{t} & =\frac{k^{2}}{T^{2}}\left\|\sum_{s=1}^{T} \tilde{F}_{s}^{k} F_{s}^{0^{\prime}} \Lambda^{0^{\prime}} e_{t} / N \sqrt{r}\right\|^{2} \\
& \leq \frac{k^{2}}{N} \frac{1}{r}\left(\frac{1}{T} \sum_{s=1}^{T}\left\|\tilde{F}_{s}^{k}\right\|^{2}\right)\left(\frac{1}{T N} \sum_{s=1}^{T}\left\|F_{s}^{0^{\prime}} \Lambda^{0^{\prime}} e_{t}\right\|^{2}\right) \\
& =O_{p}\left(k^{3} / N\right) \frac{1}{r}\left(\frac{1}{T N} \sum_{s=1}^{T}\left\|F_{s}^{0^{\prime}} \Lambda^{0^{\prime}} e_{t}\right\|^{2}\right)
\end{aligned}
$$


because $T^{-1} \sum_{s=1}^{T}\left\|\tilde{F}_{s}^{k}\right\|^{2}=O_{p}(k)$.

Next, we show that $A \stackrel{\text { def }}{=}(T N)^{-1} \sum_{s=1}^{T}\left\|F_{s}^{0^{\prime}} \Lambda^{0^{\prime}} e_{t}\right\|^{2}=O_{p}(r)$.

$$
\begin{aligned}
E(|A|) & =\frac{1}{N T} \sum_{s=1}^{T} E\left\|F_{s}^{0^{\prime}} \Lambda^{0^{\prime}} e_{t}\right\|^{2} \\
& =\frac{1}{N T} \sum_{s=1}^{T} \sum_{l=1}^{r} \sum_{m=1}^{r} \sum_{i=1}^{N} \sum_{j=1}^{N} E\left(e_{i t} e_{j t}\right) E\left(F_{s l}^{0} F_{s m}^{0}\right) \Lambda_{i l}^{0} \lambda_{j m}^{0} \\
& =\frac{1}{N T} \sum_{s=1}^{T} \sum_{l=1}^{r} \sum_{i=1}^{N} E\left(e_{i t}^{2}\right) E\left(\left(F_{s l}^{0}\right)^{2}\right)\left(\Lambda_{i l}^{0}\right)^{2} \\
& =O(r),
\end{aligned}
$$

because of the zero correlation assumptions that $E\left(e_{i t} e_{j t}\right)=0$ for $j \neq i$ and $E\left(F_{s l}^{0} F_{s m}^{0}\right)=0$ for $m \neq l$. This implies that $A=O_{p}(r)$. Hence, $c_{t}=O_{p}\left(k^{3} / N\right)$. This completes the proof of Proposition 3.2.

From the above proof we can see that the conclusion of Proposition 3.2 still holds true if the zero correlation assumptions are replaced by some weakly dependent assumptions such as $N^{-1} \sum_{i=1}^{N} \sum_{j \neq i}^{N} E\left(e_{i t} e_{j t} c_{1, i j l m}\right)=$ $O(1)$ and $r^{-1} \sum_{l=1}^{r} \sum_{m \neq l}^{r} E\left(F_{s l}^{0} F_{s m}^{0} c_{2, l m l m}\right)=O(1)$, where $c_{1, i j l m}$ and $c_{2, i j l m}$ are some bounded sequences of non-random numbers depending on $i, j, l, m$.

\section{Proof of Theorem 3.1}

Lemma 2 Let $D_{k}=\hat{F}^{k^{\prime}} \hat{F}^{k} / T$ and $D_{0}=H^{k^{\prime}} F^{0^{\prime}} F^{0} H^{k} / T$. When $k \leq r$, we have (i) $\left\|D_{k}^{-1}\right\|=O_{p}(k)$; (ii) $\left\|D_{k}^{-1}-D_{0}^{-1}\right\|=O_{p}\left(\max \left\{\frac{k_{\text {max }}^{4} r^{1.5}}{\sqrt{N}}, \frac{k_{\text {max }}^{4} r}{\sqrt{T}}\right\}\right)$.

Proof : Following Bai and $\mathrm{Ng}$ (2002), we have

$$
\begin{aligned}
D_{k}-D_{0}= & \frac{\hat{F}^{k^{\prime}} \hat{F}^{k}}{T}-\frac{H^{k^{\prime}} F^{0^{\prime}} F^{0} H^{k}}{T} \\
= & \frac{1}{T} \sum_{t=1}^{T}\left[\hat{F}_{t}^{k} \hat{F}_{t}^{k^{\prime}}-H^{k^{\prime}} F_{t}^{0} F_{t}^{0^{\prime}} H^{k}\right] \\
= & \frac{1}{T} \sum_{t=1}^{T}\left(\hat{F}_{t}^{k}-H^{k^{\prime}} F_{t}^{0}\right)\left(\hat{F}_{t}^{k}-H^{k^{\prime}} F_{t}^{0}\right)^{\prime}+\frac{1}{T} \sum_{t=1}^{T}\left(\hat{F}_{t}^{k}-H^{k^{\prime}} F_{t}^{0}\right) F_{t}^{0^{\prime}} H^{k} \\
& +\frac{1}{T} \sum_{t=1}^{T} H^{k^{\prime}} F_{t}^{0}\left(\hat{F}_{t}^{k}-H^{k^{\prime}} F_{t}^{0}\right)^{\prime} .
\end{aligned}
$$


Hence, we have

$$
\begin{aligned}
\left\|D_{k}-D_{0}\right\| & \leq \frac{1}{T} \sum_{t=1}^{T}\left\|\hat{F}_{t}^{k}-H^{k^{\prime}} F_{t}^{0}\right\|^{2}+2\left(\frac{1}{T} \sum_{t=1}^{T}\left\|\hat{F}_{t}^{k}-H^{k^{\prime}} F_{t}^{0}\right\|^{2}\right)^{1 / 2}\left(\frac{1}{T} \sum_{t=1}^{T}\left\|H^{k^{\prime}} F_{t}^{0}\right\|^{2}\right)^{1 / 2} \\
& =O_{p}\left(\max \left\{\frac{k^{3} r}{N}, \frac{k^{3}}{T}\right\}\right)+O_{p}\left(\max \left\{\frac{\sqrt{k^{3} r}}{\sqrt{N}}, \frac{\sqrt{k^{3}}}{\sqrt{T}}\right\}\right) \cdot O_{p}\left(\sqrt{k r^{2}}\right) \\
& =O_{p}\left(\max \left\{\frac{k^{2} r^{1.5}}{\sqrt{N}}, \frac{k^{2} r}{\sqrt{T}}\right\}\right)
\end{aligned}
$$

by Proposition 3.1 and the fact that $\frac{1}{T} \sum_{t=1}^{T}\left\|H^{k^{\prime}} F_{t}^{0}\right\|^{2}=O_{p}\left(k r^{2}\right)$, which is shown below.

From weakly dependent process of $F_{t}^{0}$, it is easy to show that

$$
\frac{1}{T} \sum_{t=1}^{T}\left\|H^{k^{\prime}} F_{t}^{0}\right\|^{2}-E\left[\frac{1}{T} \sum_{t=1}^{T}\left\|H^{k^{\prime}} F_{t}^{0}\right\|^{2}\right]=O_{p}\left(\frac{1}{\sqrt{T}}\right) .
$$

Also, one can easily show that $\left\|D_{k}^{-1}\right\|=O_{p}(k)$. Then from $D_{k}^{-1}-D_{0}^{-1}=D_{k}^{-1}\left(D_{0}-D_{k}\right) D_{0}^{-1}$, we have

$$
\begin{aligned}
\left\|D_{k}^{-1}-D_{0}^{-1}\right\| & =\left\|D_{k}^{-1}\left(D_{o}-D_{k}\right) D_{0}^{-1}\right\| \\
& \leq\left\|D_{k}^{-1}\right\| \cdot\left\|D_{0}-D_{k}\right\| \cdot\left\|D_{0}^{-1}\right\| \\
& =k^{2} \frac{\left\|D_{k}^{-1}\right\|}{k} \cdot\left\|D_{0}-D_{k}\right\| \cdot \frac{\left\|D_{0}^{-1}\right\|}{k} \\
& =k^{2} \cdot O_{p}(1) \cdot O_{p}\left(\max \left\{\frac{k^{2} r^{1.5}}{\sqrt{N}}, \frac{k^{2} r}{\sqrt{T}}\right\}\right) \\
& =O_{p}\left(\max \left\{\frac{k_{\text {max }}^{4} r^{1.5}}{\sqrt{N}}, \frac{k_{\text {max }}^{4} r}{\sqrt{T}}\right\}\right) .
\end{aligned}
$$

Lemma 3 For $1 \leq k \leq r$, and the $H^{k}$ defined in Proposition 3.1, we have

$$
V\left(k, \hat{F}^{k}\right)-V\left(k, F^{0} H^{k}\right)=O_{p}\left(\max \left\{\frac{k_{\max }^{5} r^{3.5}}{\sqrt{N}}, \frac{k_{\max }^{5} r^{3}}{\sqrt{T}}\right\}\right) .
$$

Proof : For the true factor matrix with $r$ factors and $H^{k}$ defined in Proposition 3.1, let $M_{F H}^{0}=I-P_{F H}^{0}$ denote the idempotent matrix spanned by null space of $F^{0} H^{k}$, with $P_{F H^{0}}=F^{0} H^{k}\left(H^{k^{\prime}} F^{0^{\prime}} F^{0} H^{k}\right)^{-1} H^{k^{\prime}} F^{0^{\prime}}$. Correspondingly, let $M_{\hat{F}}^{k}=I_{T}-\hat{F}^{k}\left(\hat{F}^{k^{\prime}} \hat{F}^{k}\right)^{-1} \hat{F}^{k^{\prime}}=I_{T}-P_{\hat{F}}^{k}$. Then

$$
\begin{aligned}
& V\left(k, \hat{F}^{k}\right)=\frac{1}{N T} \sum_{i=1}^{N} \underline{X}_{i}^{\prime} M_{\hat{F}}^{k} \underline{X}_{i}, \\
& V\left(k, F^{0} H^{k}\right)=\frac{1}{N T} \sum_{i=1}^{N} \underline{X}_{i}^{\prime} M_{F H}^{0} \underline{X}_{i}, \\
& V\left(k, \hat{F}^{k}\right)-V\left(k, F^{0} H^{k}\right)=\frac{1}{N T} \sum_{i=1}^{N} \underline{X}_{i}^{\prime}\left(P_{F H}^{0}-P_{\hat{F}}^{k}\right) \underline{X}_{i} .
\end{aligned}
$$


Following Bai and $\mathrm{Ng}(2002)$, let $D_{k}=\hat{F}^{k^{\prime}} \hat{F}^{k} / T$ and $D_{0}=H^{k^{\prime}} F^{0^{\prime}} F^{0} H^{k} / T$. Then

$$
\begin{aligned}
P_{\hat{F}}^{k}-P_{F H}^{0}= & \frac{1}{T} \hat{F}^{k}\left(\frac{\hat{F}^{k^{\prime}} \hat{F}^{k}}{T}\right)^{-1} \hat{F}^{k^{\prime}}-\frac{1}{T} F^{0} H^{k}\left(\frac{H^{k^{\prime}} F^{0^{\prime}} F^{0} H^{k}}{T}\right)^{-1} H^{k^{\prime}} F^{0^{\prime}} \\
= & \frac{1}{T}\left[\hat{F}^{k^{\prime}} D_{k}^{-1} \hat{F}^{k}-F^{0} H^{k} D_{0}^{-1} H^{k^{\prime}} F^{0^{\prime}}\right] \\
= & \frac{1}{T}\left[\left(\hat{F}^{k}-F^{0} H^{k}+F^{0} H^{k}\right) D_{k}^{-1}\left(\hat{F}^{k}-F^{0} H^{k}+F^{0} H^{k}\right)^{\prime}-F^{0} H^{k} D_{0}^{-1} H^{k^{\prime}} F^{0^{\prime}}\right] \\
= & \frac{1}{T}\left[\left(\hat{F}^{k}-F^{0} H^{k}\right) D_{k}^{-1}\left(\hat{F}^{k}-F^{0} H^{k}\right)^{\prime}+\left(\hat{F}^{k}-F^{0} H^{k}\right) D_{k}^{-1} H^{k^{\prime}} F^{0^{\prime}}\right. \\
& \left.+F^{0} H^{k} D_{k}^{-1}\left(\hat{F}^{k}-F^{0} H^{k}\right)^{\prime}-F^{0} H^{k} D_{0}^{-1} H^{k^{\prime}} F^{0^{\prime}}\right] .
\end{aligned}
$$

Thus, $N^{-1} T^{-1} \sum_{i=1}^{N} \underline{X}_{i}^{\prime}\left(P_{\hat{F}}^{k}-P_{F H}^{0}\right) \underline{X}_{i}=I+I I+I I I+I V$. We consider each term in turn.

$$
\begin{aligned}
I & =\frac{1}{N T^{2}} \sum_{i=1}^{N} \sum_{t=1}^{T} \sum_{s=1}^{T}\left(\hat{F}_{t}^{k}-H^{k^{\prime}} F_{t}^{0}\right)^{\prime} D_{k}^{-1}\left(\hat{F}_{s}^{k}-H^{k^{\prime}} F_{s}^{0}\right) X_{i t} X_{i s} \\
& \leq\left(\frac{1}{T^{2}} \sum_{t=1}^{T} \sum_{s=1}^{T}\left(\hat{F}_{t}^{k}-H^{k^{\prime}} F_{t}^{0}\right)^{\prime} D_{k}^{-1}\left(\hat{F}_{s}^{k}-H^{k^{\prime}} F_{s}^{0}\right)\right)^{1 / 2} \cdot\left(\frac{1}{T^{2}} \sum_{t=1} \sum_{s=1}\left(\frac{1}{N} \sum_{i=1}^{N} X_{i t} X_{i s}\right)^{2}\right)^{1 / 2} \\
& \leq\left(\frac{1}{T} \sum_{t=1}^{T}\left\|\hat{F}_{t}^{k}-H^{k^{\prime}} F_{t}^{0}\right\|^{2}\right) \cdot\left\|D_{k}^{-1}\right\| \cdot O_{P}(1) \\
& =O_{p}\left(\max \left\{\frac{k^{3} r}{N}, \frac{k^{3}}{T}\right\}\right) \cdot k \cdot O_{p}(1) \\
& =O_{p}\left(\max \left\{\frac{k^{4} r}{N}, \frac{k^{4}}{T}\right\}\right)
\end{aligned}
$$

by Proposition 3.1, Lemma 1(iii) and Lemma 2(i).

$$
\begin{aligned}
I I & =\frac{1}{N T^{2}} \sum_{i=1}^{N} \sum_{t=1}^{T} \sum_{s=1}^{T}\left(\hat{F}_{t}^{k}-H^{k^{\prime}} F_{t}^{0}\right)^{\prime} D_{k}^{-1} H^{k^{\prime}} F_{s}^{0} X_{i t} X_{i s} \\
& \leq\left(\frac{1}{T^{2}} \sum_{t=1}^{T} \sum_{s=1}^{T}\left\|\hat{F}_{t}^{k}-H^{k^{\prime}} F_{t}^{0}\right\|^{2} \cdot\left\|H^{k^{\prime}} F_{s}^{0}\right\|^{2} \cdot\left\|D_{k}^{-1}\right\|^{2}\right)^{1 / 2} \cdot\left(\frac{1}{T^{2}} \sum_{t=1} \sum_{s=1}\left(\frac{1}{N} \sum_{i=1}^{N} X_{i t} X_{i s}\right)^{2}\right)^{1 / 2} \\
& \leq\left(\frac{1}{T} \sum_{t=1}^{T}\left\|\hat{F}_{t}^{k}-H^{k^{\prime}} F_{t}^{0}\right\|^{2}\right)^{1 / 2} \cdot\left\|D_{k}^{-1}\right\| \cdot\left(\frac{k r^{2}}{T k r^{2}} \sum_{s=1}^{T}\left\|H^{k^{\prime}} F_{s}^{0}\right\|^{2}\right)^{1 / 2} \cdot O_{p}(1) \\
& =O_{p}\left(\max \left\{\left(\frac{k^{3} r}{N}\right)^{1 / 2},\left(\frac{k^{3}}{T}\right)^{1 / 2}\right\}\right) \cdot k \cdot k^{1 / 2} r \cdot O_{p}(1) \\
& =O_{p}\left(\max \left\{\frac{k^{3} r^{1.5}}{\sqrt{N}}, \frac{k^{3} r}{\sqrt{T}}\right\}\right) .
\end{aligned}
$$

Similarly, one can verify that $I I I$ is also $O_{p}\left(\max \left\{\frac{k^{3} r^{1.5}}{\sqrt{N}}, \frac{k^{3} r}{\sqrt{T}}\right\}\right)$. 


$$
\begin{aligned}
I V & =\frac{1}{N T^{2}} \sum_{i=1}^{N} \sum_{t=1}^{T} \sum_{s=1}^{T} F_{t}^{0^{\prime}} H^{k}\left(D_{k}^{-1}-D_{0}^{-1}\right) H^{k^{\prime}} F_{s}^{0} X_{i t} X_{i s} \\
& \leq\left\|D_{k}^{-1}-D_{0}^{-1}\right\| \frac{1}{N} \sum_{i=1}^{N}\left(\frac{1}{T} \sum_{t=1}^{T}\left\|H^{k^{\prime}} F_{t}^{0}\right\| \cdot\left|X_{i t}\right|\right)^{2} \\
& \leq\left\|D_{k}^{-1}-D_{0}^{-1}\right\| \frac{k r^{2}}{N} \sum_{i=1}^{N}\left(\frac{1}{T \sqrt{k} r} \sum_{t=1}^{T}\left\|H^{k^{\prime}} F_{t}^{0}\right\|\right)^{2} \\
& =\left\|D_{k}^{-1}-D_{0}^{-1}\right\| \cdot k r^{2} \cdot O_{p}(1) \\
& =O_{p}\left(\max \left\{\frac{k^{4} r^{4.5}}{\sqrt{N}}, \frac{k^{4} r^{4}}{\sqrt{T}}\right\}\right),
\end{aligned}
$$

where we used $\left\|D_{k}^{-1}-D_{0}^{-1}\right\|=O_{p}\left(\max \left\{\frac{k^{3} r^{2.5}}{\sqrt{N}}, \frac{k^{3} r^{2}}{\sqrt{T}}\right\}\right)$ by Lemma 2 (ii).

Thus, we have

$$
V\left(k, \hat{F}^{k}\right)-V\left(k, F^{0} H^{k}\right)=O_{p}\left(\max \left\{\frac{k_{\max }^{5} r^{3.5}}{\sqrt{N}}, \frac{k_{\max }^{5} r^{3}}{\sqrt{T}}\right\}\right)
$$

Lemma 4 For the matrix $H^{k}$ defined in Proposition 3.1, and for each $k$ with $k<r=r_{N, T} \rightarrow \infty$, there exists a positive constant $\mathrm{C}$ such that

$$
\operatorname{plim} \inf _{N, T \rightarrow \infty} \inf _{k}\left[V\left(k, F^{0} H^{k}\right)-V\left(r, F^{0}\right)\right] \geq C>0 .
$$

\section{Proof :}

$$
\begin{aligned}
V\left(k, F^{0} H^{k}\right)-V\left(r, F^{0}\right)= & \frac{1}{N T} \sum_{i=1}^{N} \underline{X}_{i}^{\prime} M_{F H}^{0} \underline{X}_{i}-\frac{1}{N T} \sum_{i=1}^{N} \underline{X}_{i}^{\prime} M_{F}^{0} \underline{X}_{i} \\
= & \frac{1}{N T} \sum_{i=1}^{N}\left(\frac{1}{\sqrt{r}} F^{0} \lambda_{i}^{0}+\underline{e}_{i}\right)^{\prime} M_{F H}^{0}\left(\frac{1}{\sqrt{r}} F^{0} \lambda_{i}^{0}+\underline{e}_{i}\right)-\frac{1}{N T} \sum_{i=1}^{N} \underline{e}_{i}^{\prime} M_{F}^{0} \underline{e}_{i} \\
= & \frac{1}{N T r} \sum_{i=1}^{N} \lambda_{i}^{0^{\prime}} F^{0^{\prime}} M_{F H}^{0} F^{0} \lambda_{i}^{0}+\frac{2}{N T \sqrt{r}} \sum_{i=1}^{N} \underline{e}_{i}^{\prime} M_{F H}^{0} F^{0} \lambda_{i}^{0} \\
& +\frac{1}{N T} \sum_{i=1}^{N} \underline{e}_{i}^{\prime}\left(P_{F}^{0}-P_{F H}^{0}\right) \underline{e}_{i} \\
= & A+B+D .
\end{aligned}
$$

Notice that $P_{F}^{0}-P_{F H}^{0} \geq 0$, thus $I I I \geq 0$. For the first term,

$$
\begin{aligned}
A & =\frac{1}{N T r} \sum_{i=1}^{N} \lambda_{i}^{0^{\prime}} F^{0^{\prime}} M_{F H}^{0} F^{0} \lambda_{i}^{0} \\
& =\frac{1}{N T r} \sum_{i=1}^{N}\left(M_{F H}^{0} F^{0} \lambda_{i}^{0}\right)^{\prime} M_{F H}^{0} F^{0} \lambda_{i}^{0} \\
& \geq C>0
\end{aligned}
$$


because $k<r$ and $M_{F H}^{0} F^{0} \lambda_{i}^{0} \neq 0$.

Next,

$$
B=\frac{2}{N T \sqrt{r}} \sum_{i=1}^{N} \underline{e}_{i}^{\prime} F^{0} \lambda_{i}^{0}-\frac{2}{N T \sqrt{r}} \sum_{i=1}^{N} \underline{e}_{i}^{\prime} P_{F H}^{0} F^{0} \lambda_{i}^{0} .
$$

Consider the first term

$$
\begin{aligned}
\left|\frac{1}{N T \sqrt{r}} \sum_{i=1}^{N} \underline{e}_{i}^{\prime} F^{0} \lambda_{i}^{0}\right| & =\left|\frac{1}{N T \sqrt{r}} \sum_{i=1}^{N} \sum_{t=1}^{T} \underline{e}_{i t} F_{t}^{0^{\prime}} \lambda_{i}^{0}\right| \\
& \leq\left(\frac{1}{T r} \sum_{t=1}^{T}\left\|F_{t}^{0}\right\|^{2}\right)^{1 / 2} \cdot \sqrt{r} \cdot \frac{1}{\sqrt{N}}\left(\frac{1}{T} \sum_{t=1}^{T}\left\|\frac{1}{\sqrt{N r}} \sum_{i=1}^{N} e_{i t} \lambda_{i}^{0}\right\|^{2}\right)^{1 / 2} \\
& =O_{p}\left(\frac{\sqrt{r}}{\sqrt{N}}\right)
\end{aligned}
$$

where the last equality follows from assumption $\mathrm{C} 2$. The second term is also $o_{p}(1)$, and hence $B=o_{p}(1)$.

Lemma 5 For any $k$ with $r \leq k \leq k_{\max }, V\left(k, \hat{F}^{k}\right)-V\left(r, \hat{F}^{r}\right)=O_{p}\left(\max \left\{\frac{k_{\max }^{2} r}{N}, \frac{k_{\max }^{2} r^{0.5}}{T}\right\}\right)$.

Proof :

$$
\begin{aligned}
\left|V\left(k, \hat{F}^{k}\right)-V\left(r, \hat{F}^{r}\right)\right| & \leq\left|V\left(k, \hat{F}^{k}\right)-V\left(r, F^{0}\right)\right|+\left|V\left(r, F^{0}\right)-V\left(r, \hat{F}^{r}\right)\right| \\
& \leq 2 \max _{r \leq k}\left|V\left(k, \hat{F}^{k}\right)-V\left(r, F^{0}\right)\right| .
\end{aligned}
$$

Thus, it is sufficient to prove for each $k$ with $r \leq k \leq k_{\max }$,

$$
V\left(k, \hat{F}^{k}\right)-V\left(r, F^{0}\right)=O_{p}\left(\max \left\{\frac{k_{\max } r^{2}}{\sqrt{N}}, \frac{k_{\max } r^{1.5}}{\sqrt{T}}\right\}\right) .
$$

Let $H^{k}$ be as defined in Proposition 3.1, with full row rank. Let the $k \times r$ matrix $H^{k+}$ be the generalized inverse of $H^{k}$ such that $H^{k} H^{k+}=I_{r}$. From $\underline{X}_{i}=\frac{1}{\sqrt{r}} F^{0} \lambda_{i}^{0}+\underline{e}_{i}$, we have $\underline{X}_{i}=\frac{1}{\sqrt{r}} F^{0} H^{k} H^{k+} \lambda_{i}^{0}+\underline{e}_{i}$. This implies that

$$
\begin{aligned}
\underline{X}_{i} & =\frac{1}{\sqrt{r}} \hat{F}^{k} H^{k+} \lambda_{i}^{0}+\underline{e}_{i}-\frac{1}{\sqrt{r}}\left(\hat{F}^{k}-F^{0} H^{k}\right) H^{k+} \lambda_{i}^{0} \\
& =\frac{1}{\sqrt{r}} \hat{F}^{k} H^{k+} \lambda_{i}^{0}+\underline{u}_{i},
\end{aligned}
$$

where $\underline{u}_{i}=\underline{e}_{i}-\frac{1}{\sqrt{r}}\left(\hat{F}^{k}-F^{0} H^{k}\right) H^{k+} \lambda_{i}^{0}$. 
Note that

$$
\begin{aligned}
V\left(k, \hat{F}^{k}\right)= & \frac{1}{N T} \sum_{i=1}^{N} \underline{u}_{i}^{\prime} M_{\hat{F}}^{k} \underline{u}_{i}, \\
V\left(r, F^{0}\right)= & \frac{1}{N T} \sum_{i=1}^{N} \underline{e}_{i}^{\prime} M_{F}^{0} \underline{e}_{i}, \\
V\left(k, \hat{F}^{k}\right)= & \frac{1}{N T} \sum_{i=1}^{N}\left(\underline{e}_{i}-\frac{1}{\sqrt{r}}\left(\hat{F}^{k}-F^{0} H^{k}\right) H^{k+} \lambda_{i}^{0}\right)^{\prime} M_{\hat{F}}^{k}\left(\underline{e}_{i}-\frac{1}{\sqrt{r}}\left(\hat{F}^{k}-F^{0} H^{k}\right) H^{k+} \lambda_{i}^{0}\right), \\
= & \frac{1}{N T} \sum_{i=1}^{N} \underline{e}_{i}^{\prime} M_{\hat{F}}^{k} \underline{e}_{i}-\frac{2}{N T \sqrt{r}} \sum_{i=1}^{N} \lambda_{i}^{0^{\prime}} H^{k+^{\prime}}\left(\hat{F}^{k}-F^{0} H^{k}\right)^{\prime} M_{\hat{F}}^{k} \underline{e}_{i} \\
& +\frac{1}{N T r} \sum_{i=1}^{N} \lambda_{i}^{0^{\prime}} H^{k+{ }^{\prime}}\left(\hat{F}^{k}-F^{0} H^{k}\right)^{\prime} M_{\hat{F}}^{k}\left(\hat{F}^{k}-F^{0} H^{k}\right) H^{k+} \lambda_{i}^{0} \\
= & a+b+c .
\end{aligned}
$$

Because $I-M_{\hat{F}}^{k}$ is positive semi-definite, $x^{\prime} M_{\hat{F}}^{k} x \leq x^{\prime} x$. Thus

$$
\begin{aligned}
c & \leq \frac{1}{N T r} \sum_{i=1}^{N} \lambda_{i}^{0^{\prime}} H^{k+^{\prime}}\left(\hat{F}^{k}-F^{0} H^{k}\right)^{\prime}\left(\hat{F}^{k}-F^{0} H^{k}\right) H^{k+} \lambda_{i}^{0} \\
& \leq \frac{1}{T} \sum_{t=1}^{T}\left\|\hat{F}_{t}^{k}-H^{k^{\prime}} F_{t}^{0}\right\|^{2} \cdot\left(\frac{1}{N r} \sum_{i=1}^{N}\left\|\lambda_{i}^{0}\right\|^{2}\left\|H^{k+}\right\|^{2}\right) \\
& =O_{p}\left(\max \left\{\frac{k^{3} r}{N}, \frac{k^{3}}{T}\right\}\right) \cdot O_{p}(k r) \\
& =O_{p}\left(\max \left\{\frac{k^{4} r^{2}}{N}, \frac{k^{4} r}{T}\right\}\right)
\end{aligned}
$$

by Proposition 3.1 .

For term $b$, we use the fact that $|\operatorname{tr}(A)| \leq r\|A\|$ for any $r \times r$ matrix $A$. Thus

$$
\begin{aligned}
b & =\frac{2}{T \sqrt{r}} \operatorname{tr}\left(H^{k+}\left(\hat{F}^{k}-F^{0} H^{k}\right)^{\prime} M_{\hat{F}}^{k}\left(\frac{1}{N} \sum_{i=1}^{N} \underline{e}_{i} \lambda_{i}^{0}\right)\right) \\
& \leq 2 \cdot\left\|H^{k+}\right\| \cdot\left\|\frac{\hat{F}^{k}-F^{0} H^{k}}{\sqrt{T}}\right\| \cdot\left\|\frac{1}{\sqrt{T r} N} \sum_{i=1}^{N} \underline{e}_{i} \lambda_{i}^{0}\right\| \\
& \leq 2 \cdot\left\|H^{k+}\right\| \cdot\left(\frac{1}{T} \sum_{t=1}^{T}\left\|\hat{F}^{k}-F^{0} H^{k}\right\|^{2}\right)^{1 / 2} \cdot \frac{1}{\sqrt{N}}\left(\frac{1}{T} \sum_{t=1}^{T}\left\|\frac{1}{\sqrt{N r}} \sum_{i=1} \underline{e}_{i} \lambda_{i}^{0}\right\|^{2}\right)^{1 / 2} \\
& =2 \cdot(k r)^{1 / 2} \cdot O_{p}\left(\max \left\{\frac{\sqrt{k^{3} r}}{\sqrt{N}}, \frac{\sqrt{k^{3}}}{\sqrt{T}}\right\}\right) \cdot O_{p}(1) \\
& =O_{p}\left(\max \left\{\frac{k^{2} r}{\sqrt{N}}, \frac{k^{2} r^{0.5}}{\sqrt{T}}\right\}\right)
\end{aligned}
$$

by Proposition 3.1 and assumption C2. Therefore,

$$
V\left(k, \hat{F}^{k}\right)=\frac{1}{N T} \sum_{i=1}^{N} \underline{e}_{i}^{\prime} M_{\hat{F}}^{k} \underline{e}_{i}+O_{p}\left(\max \left\{\frac{k^{2} r}{\sqrt{N}}, \frac{k^{2} r^{0.5}}{\sqrt{T}}\right\}\right) .
$$


Thus we have

$$
V\left(k, \hat{F}^{k}\right)-V\left(r, F^{0}\right)=\frac{1}{N T} \sum_{i=1}^{N} \underline{e}_{i}^{\prime} P_{F}^{0} \underline{e}_{i}-\frac{1}{N T} \sum_{i=1}^{N} \underline{e}_{i}^{\prime} P_{\hat{F}}^{k} \underline{e}_{i}+O_{p}\left(\max \left\{\frac{k^{2} r}{\sqrt{N}}, \frac{k^{2} r^{0.5}}{\sqrt{T}}\right\}\right) .
$$

Note that

$$
\begin{aligned}
\frac{1}{N T} \sum_{i=1}^{N} \underline{e}_{i}^{\prime} P_{F}^{0} \underline{e}_{i} & \leq\left\|\left(\frac{F^{0^{\prime}} F^{0}}{T}\right)^{-1}\right\| \cdot \frac{1}{N T^{2}} \sum_{i=1}^{N} \underline{e}_{i}^{\prime} F^{0} F^{0^{\prime}} \underline{e}_{i} \\
& =\left\|\left(\frac{F^{0^{\prime}} F^{0}}{T}\right)^{-1}\right\| \cdot \frac{1}{N T} \sum_{i=1}^{N}\left\|\frac{1}{\sqrt{T r}} \sum_{t=1}^{T} F_{t}^{0} \underline{e}_{i t}\right\|^{2} \cdot r \\
& =r \cdot O_{p}(1) \cdot \frac{1}{T} \cdot r \cdot O_{p}(1) \\
& =O_{p}\left(\frac{r^{2}}{T}\right) \leq O_{p}\left(\max \left\{\frac{k^{2} r}{\sqrt{N}}, \frac{k^{2} r^{0.5}}{\sqrt{T}}\right\}\right) .
\end{aligned}
$$

$\frac{1}{N T} \sum_{i=1}^{N} \underline{e}_{i}^{\prime} P_{F}^{0} \underline{e}_{i}$ is bounded by the sum of the first $k$ largest eigenvalues of the matrix $A_{N T}=\frac{1}{N T} e^{\prime} e$, where $e=\left(e_{t i}\right), T \times N$. Let $\rho(A)$ denote the largest eigenvalue of a matrix $A$. Under Assumption B6, as Bai and $\operatorname{Ng}(2005)$ shows, $\rho\left(A_{N T}\right)=O_{p}\left(C_{N T}^{-2}\right)$, where $C_{N T}^{2}=\min \{N, T\}$. Thus,

$$
\frac{1}{N T} \sum_{i=1}^{N} \underline{e}_{i}^{\prime} P_{F}^{0} \underline{e}_{i}=O_{p}\left(\max \left\{\frac{k}{N}, \frac{k}{T}\right\}\right) \leq O_{p}\left(\max \left\{\frac{k^{2} r}{\sqrt{N}}, \frac{k^{2} r^{0.5}}{\sqrt{T}}\right\}\right) .
$$

In summary, we have shown that

$$
V\left(k, \hat{F}^{k}\right)-V\left(r, F^{0}\right)=O_{p}\left(\max \left\{\frac{k_{\max }^{2} r}{\sqrt{N}}, \frac{k_{\max }^{2} r^{0.5}}{\sqrt{T}}\right\}\right)
$$

\section{Proof of Theorem 3.1}

Proof : We shall prove that $\lim _{N, T \rightarrow \infty} P(P C(k)<P C(r))=0$ for all $k \neq r$. Since

$$
P C(k)-P C(r)=V\left(k, \hat{F}^{k}\right)-V\left(r, \hat{F}^{r}\right)-(r-k) g(N, T),
$$

it is sufficient to prove that $P\left[V\left(k, \hat{F}^{k}\right)-V\left(r, \hat{F}^{r}\right)<(r-k) g(N, T)\right] \rightarrow 0$ as $N, T, k, r \rightarrow \infty$.

Consider $k<r$. We have the identity:

$$
\begin{aligned}
V\left(k, \hat{F}^{k}\right)-V\left(r, \hat{F}^{r}\right)= & {\left[V\left(k, \hat{F}^{k}\right)-V\left(k, F^{0} H^{k}\right)\right]+\left[V\left(k, F^{0} H^{k}\right)-V\left(r, F^{0} H^{r}\right)\right] } \\
& +\left[V\left(r, F^{0} H^{r}\right)-V\left(r, \hat{F}^{r}\right)\right] .
\end{aligned}
$$

Lemma 3 implies that the first and the third terms are both $O_{p}\left(\max \left\{\frac{k_{\max }^{8.5}}{\sqrt{N}}, \frac{k_{\max }^{8}}{\sqrt{T}}\right\}\right)$. Next, we consider the second item. Because $F^{0} H^{r}$ and $F^{0}$ span the same column space, $V\left(r, F^{0} H^{r}\right)=V\left(r, F^{0}\right)$. Thus the 
second item can be rewritten as $V\left(k, F^{0} H^{k}\right)-V\left(r, F^{0}\right)$, which has a positive limit by Lemma 4 . Hence $P[P C(k)<P C(r)] \rightarrow 0$ if $(r-k) g(N, T) \rightarrow 0$ as $N, T, k, r \rightarrow \infty$.

Next, for $k \geq r$,

$$
P[P C(k)-P C(r)<0]=P\left[V\left(r, \hat{F}^{r}\right)-V\left(k, \hat{F}^{k}\right)>(k-r) g(N, T)\right]
$$

By Lemma $5, V\left(r, \hat{F}^{r}\right)-V\left(k, \hat{F}^{k}\right)=O_{p}\left(\max \left\{\frac{k_{\max }^{3}}{\sqrt{N}}, \frac{k_{m a x}^{2.5}}{\sqrt{T}}\right\}\right)$. According to our setting, $(k-r) g(N, T)$ converges to zero at a slower rate than $O_{p}\left(\max \left\{\frac{k_{\text {max }}^{3}}{\sqrt{N}}, \frac{k_{\text {max }}^{2.5}}{\sqrt{T}}\right\}\right)$. Thus, for $k>r, P[P C(k)<P C(r)] \rightarrow 0$ as $N, T, k, r \rightarrow \infty$. 


\section{Appendix B: Figures}

Figure 1: Sensitivity of $P C_{p 1}$ Criterion to $k_{\max }: 200 / 60$ case

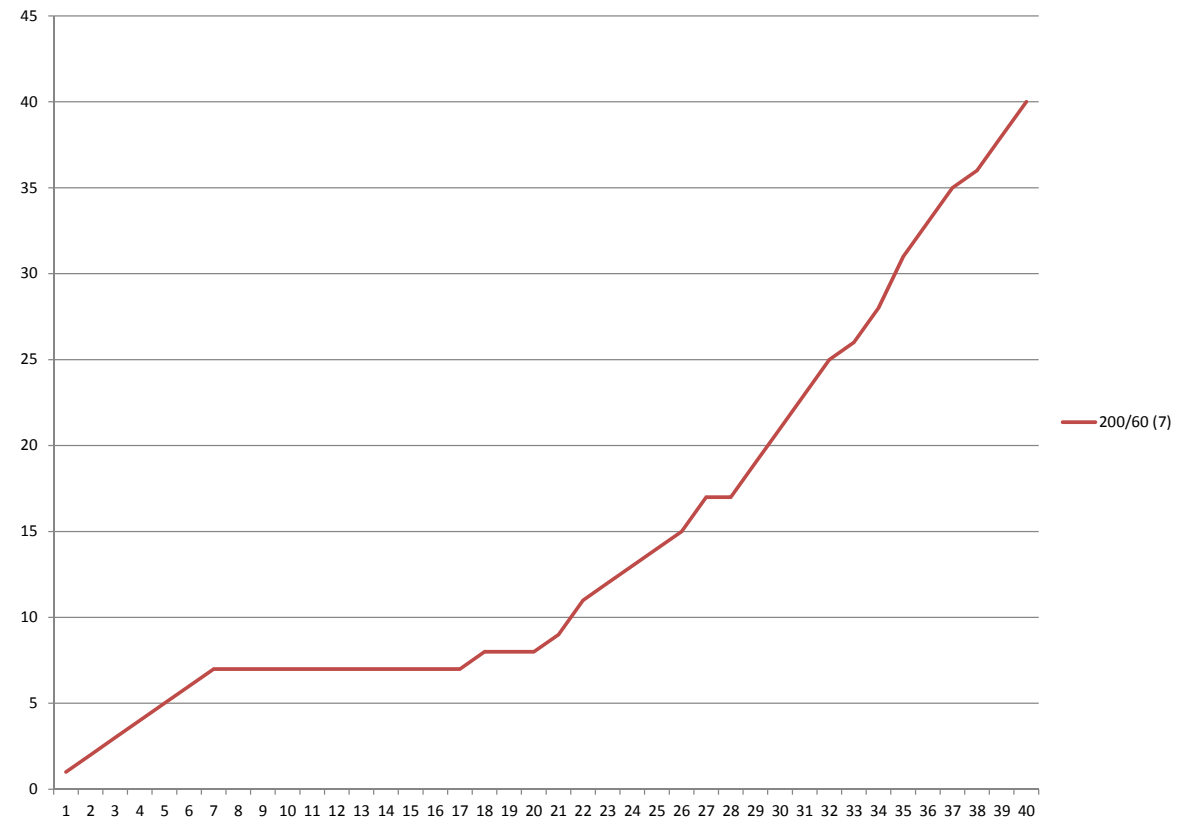

Note:The values of $\hat{k}$ estimated by $P C_{p 1}$ for $N=200, T=60$ and $r=7$ with $k_{\max } \in[1,40]$. 
Figure 2: Sensitivity of $P C_{p 1}$ Criterion to $k_{\max }$

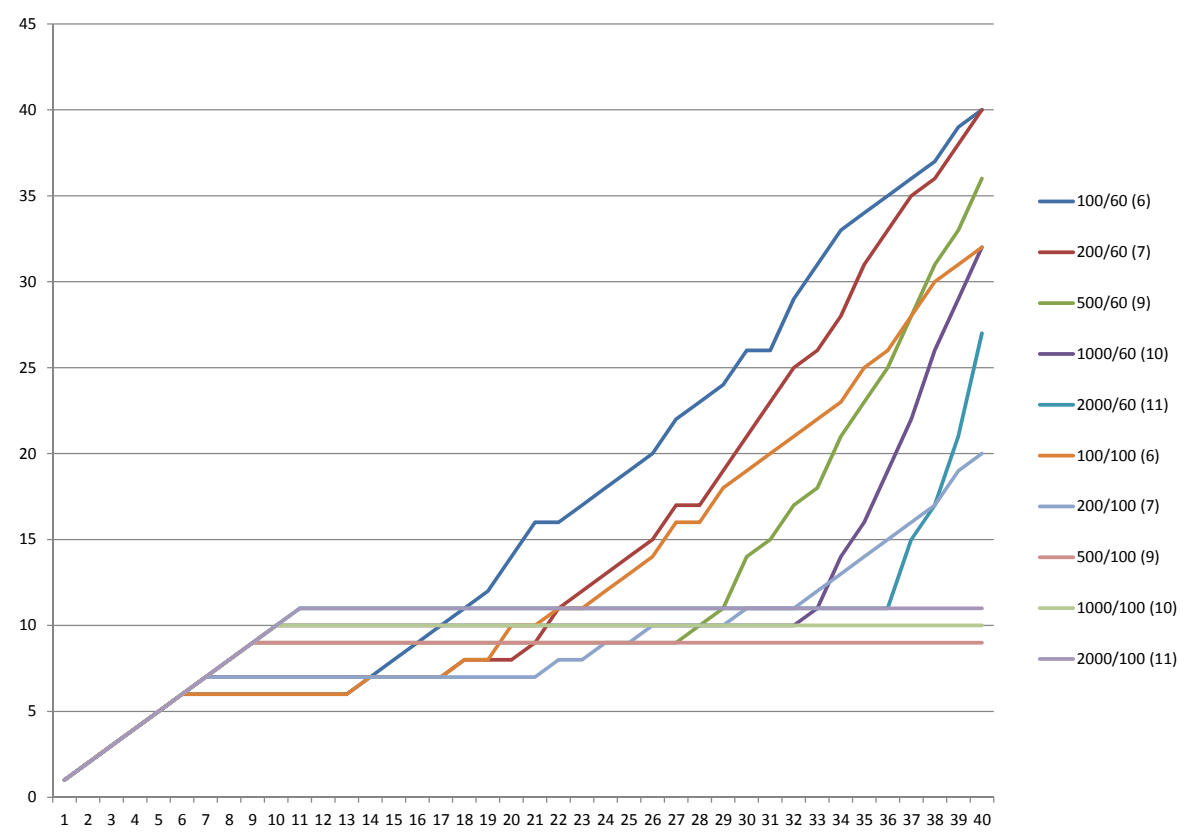

Note: Each line represents $\hat{k}$ estimated by $P C_{p 1}$ for each case of different sample size. The notation in the graph shows the sample size and the true number of factors for each case. For example, 100/60(6) means that $N=100, T=60$ and $r=6$. 


\section{References}

[1] Ahn, S., Horenstein, A., 2013. Eigenvalue Ratio Test for the Number of Factors. Econometrica 81, 12031127.

[2] Alessi, L., Barigozzi, M., Capasso, M., 2010. Improved penalization for determining the number of factors in approximate factor models. Statistics and Probability Letters 80, 1806-1813.

[3] Bai, C., Li, Q., Ouyang, M., 2014. Property Taxes and Home Prices: A Tale of Two Cities. Journal of Econometrics 180, 1-15.

[4] Bai, J., Ng, S., 2002. Determining the Number of Factors in Approximate Factor Models. Econometrica 70, 191-221.

[5] Ching, H.S., Hsiao, C., Wan, S.K., 2012. Impact of CEPA on the labor market of Hong Kong. China Economic Review 23, 975-981.

[6] Connor, G., Korajzcyk, R., 1993. A Test for the Number of Factors in an Approximate Factor Model. Journal of Finance 48, 1263-1291.

[7] Cragg, J., Donald, S., 1997. Inferring the Rank of a Matrix. Journal of Econometrics 76, 223-250.

[8] Donald, S. 1997. Inference Concerning the Number of Factors in a Multivariate Nonparameteric Relationship. Econometrica 65, 103-132.

[9] Fan, J., Liao, Y., Mincheva, M., 2011. High-Dimensional Covariance Matrix Estimation in Approximate Factor Models. Annals of Statistics 39, 3320-3356.

[10] Fan, J., Liao, Y., Mincheva, M., 2013. Large Covariance Estimation by Thresholding Principal Orthogonal Complements. Journal of the Royal Statistical Society: Series B 75, 603-680.

[11] Forni, M., Hallin, M., Lippi, M., Reichlin, L., 2000. Reference Cycles: The NBER Methodology Revisited. CEPR Discussion Paper 2400.

[12] Forni, M., Gambetti, L., 2010. Macroeconomic Shocks and the Business Cycle: Evidence from a Structural Factor Model fiscal. Working Papers 440, Barcelona Graduate School of Economics.

[13] Forni, M., Reichlin, L., 1998. Lets Get Real: a Factor-Analytic Approach to Disaggregated Business Cycle Dynamics. Review of Economic Studies 65, 453-473.

[14] Giannone, D., Reichlin L., Sala, L., 2005. Monetary Policy in Real Time. NBER Macroeconomics Annual 2004 e.d. by Mark Gertler and Kenneth Rogoff, 161-200. MIT Press. 
[15] Gregory, A., Head, A., 1999. Common and Country-Specific Fluctuations in Productivity, Investment, and the Current Account. Journal of Monetary Economics 44, 423-452.

[16] Hallin, M., Liska, R., 2007. Determining the Number of Factors in the General Dynamic Factor Model. Journal of American Statistical Association 102, 603-617.

[17] Hsiao, C., Ching, H.S., Wan, S.K., 2012. A Panel Data Approach for Program Evaluation: Measuring the Benefits of Political and Economic Integration of Hong Kong with Mainland China. Journal of Applied Econometrics 27, 705-740.

[18] Jurado, K., Ludvigson, S., Ng, S., 2013. Measuring Uncertainty. Working paper.

[19] Lewbel, A., 1991. The Rank of Demand Systems: Theory and Nonparametric Estimation. Econometrica $59,711-730$.

[20] Ludvigson, S., Ng, S., 2007. The empirical risk-return relation: a factor analysis approach. Journal of Financial Economics 83, 171-222.

[21] Ludvigson, S., Ng, S.,2009. Macro Factors in Bond Risk Premia. The Review of Financial Studies 22, $5027-5067$

[22] Ludvigson, S., Ng, S., 2010. A Factor Analysis of Bond Risk Premia. Handbook of Empirical Economics and Finance, e.d. by Aman Uhla and David E. A. Giles, 313-372. Chapman and Hall, Boca Raton, FL.

[23] Onatski, A., 2009. Testing Hypotheses about the Number of Factors in Large Factor Models. Econometrica $77,1447-1479$.

[24] Onatski, A., 2010. Determing the Number of Factors from Empirical Distribution of Eigenvalues. Review of Economics and Statistics 92, 1004-1016.

[25] Ouyang, M., Peng, Y., 2015. The treatment-effect estimation: A case study of the 2008 economic stimulus package of China. Journal of Econometrics 188, 545-557.

[26] Ross, S., 1976. The Arbitrage Theory of Capital Asset Pricing. Journal of Finance 13, 341-360.

[27] Stock, J. H., Watson, M., 1998. Diffusion Indexes. NBER Working Paper 6702.

[28] Stock, J. H., Watson, M., 1999. Forecasting Inflation. Journal of Monetary Economics 44, 293-335. 